\title{
Micro-macro decomposition based asymptotic-preserving numerical schemes and numerical moments conservation for collisional nonlinear kinetic equations *
}

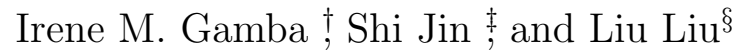

\begin{abstract}
In this paper, we first extend the micro-macro decomposition method for multiscale kinetic equations from the BGK model to general collisional kinetic equations, including the Boltzmann and the Fokker-Planck Landau equations. The main idea is to use a relation between the (numerically stiff) linearized collision operator with the nonlinear quadratic ones, the latter's stiffness can be overcome using the BGK penalization method of Filbet and Jin for the Boltzmann, or the linear Fokker-Planck penalization method of Jin and Yan for the Fokker-Planck Landau equations. Such a scheme allows the computation of multiscale collisional kinetic equations efficiently in all regimes, including the fluid regime in which the fluid dynamic behavior can be correctly computed even without resolving the small Knudsen number. A distinguished feature of these schemes is that although they contain implicit terms, they can be implemented explicitly. These schemes preserve the moments (mass, momentum and energy) exactly thanks to the use of the macroscopic system which is naturally in a conservative form. We further utilize this conservation property for more general kinetic systems, using the Vlasov-Ampère and Vlasov-Ampère-Boltzmann systems as examples. The main idea is to evolve both the kinetic equation for the probability density distribution and the moment system, the later naturally induces a scheme that conserves exactly the moments numerically if they are physically conserved.
\end{abstract}

keywords: Boltzmann equation, Landau equation, micro-macro decomposition, asymptotic preserving scheme, conservative scheme, Vlasov-Ampère-Boltzmann

\section{Introduction}

The Boltzmann equation and the Fokker-Planck-Landau equation are among the most important kinetic equations, arising in describing the dynamics of probability density distribution of particles in rarified gas and plasma, respectively. One of the main computational

*The first and the third author was supported by the funding DOE-Simulation Center for Runaway Electron Avoidance and Mitigation, project No. de-sc0016283 The second author was supported by NSF grants DMS1522184 and DMS-1107291: RNMS KI-Net, and NSFC grants No. 31571071 and No. 11871297.

${ }^{\dagger}$ Department of Mathematics and The Institute for Computational Engineering and Sciences (ICES), University of Texas at Austin, Austin, TX 78712, USA (gamba@math.utexas.edu).

${ }^{\ddagger}$ School of Mathematical Sciences, Institute of Natural Sciences, MOE-LSC and SHL-MAC, Shanghai Jiao Tong University, Shanghai, China (shijin-m@sjtu.edu.cn).

$\S$ The Institute for Computational Engineering and Sciences (ICES), University of Texas at Austin, Austin, TX 78712, USA (lliu@ices.utexas.edu). 
challenges for these kinetic equations is that the problem may often encounter multiple time and spatial scales, characterized by the Knudsen number (denoted by $\varepsilon$ ), the dimensionless mean free path, that may vary in orders of magnitude in the computational domain, covering the regimes from fluid, transition, rarefied to even free streaming regimes. AsymptoticPreserving (AP) schemes, which mimics the asymptotic transition from one scale to another at the discrete level, have been shown to be an effective computational paradigm in the last two decades [12, 13. Such schemes allow efficient numerical approximations in all regimes, and coarse mesh and large time steps can be used even in the fluid dynamic regime, without numerically resolving the small Knudsen number. For the space inhomogeneous Boltzmann equation, AP schemes were first designed using BGK-operator based penalty [6]. Other approaches include the exponential integrator based methods [5, 21, or micro-macro (MM) decomposition [2]. We also mention relevant works [33, 23, 28, One should note that [2, 33] only dealt with the BGK model, rather than the full Boltzmann equation. For AP schemes to deal with the stiff Landau collision operator, the BGK-penalization method was extended to the Fokker-Planck-Landau equation in 15], using the linear Fokker-Planck operator as the penalty.

The aim of this paper is not on the comparison of all these different approaches, rather we will focus on the micro-macro decomposition method, which was formulated in [2] for the Boltzmann but numerically realized only for the BGK model. One of the difficulties in this formulation is that one encounters a stiff linearized collision operator whose inversion could be computationally inefficient. In 18, a linear penalty for the microscopic deviation equation was used to remove the stiffness. This idea is suitable for the Boltzmann equation but not for the Fokker-Planck-Landau equation which has second derivative terms in the collision operators. One of the goals of the current paper is to show how the micro-macro decomposition method in 2] can be extended to the general collision operators, include the Boltzmann and Landau collision operators. Having its theoretical origin in [26] (see also [25]), the micro-macro decomposition has also found its advantage in designing AP schemes for radiative heat transfer [16], linear transport equation [19], among others. For the Boltzmann or the Fokker-Planck-Landau equation, the MM method is based on a decomposition of the kinetic equation under study into a coupled system composed of a kinetic equation on the microscopic part and a fluid equation on the macroscopic part. By using an implicitexplicit (IMEX) temporal discretization, it naturally leads to an AP scheme at the level of the compressible Navier-Stokes asymptotics [2]. Moreover, the MM formulation guarantees the preservation of numerical moments (mass, momentum and energy) exactly thanks to the macroscopic system which is naturally in a conservative form. Another advantage of the MM approach is that one can obtain good uniform numerical stability result [24, 10].

Our main idea for the MM method is the usage of a simple relation between a linearized collision operator (a numerically stiff term) and the quadratically nonlinear collision operator. For the latter (stiff) nonlinear collision operators, we then use the BGK-penalty method of Filbet-Jin [6] for the Boltzmann collision or the Fokker-Planck penalty of Jin-Yan [15] for the Fokker-Planck-Landau collision. This allows us to extend the MM method of 2 from the BGK model to the more physical Boltzmann and Fokker-Planck-Landau equations in a rather simple fashion.

We would like to emphasize that in the MM formalism (as well as in the penalty methods in [6. 15]), one needs to solve the macroscopic system, which is in a conservation form, giving rise the conservation of mass, momentum and total energy. When discretizing the macroscopic system with a standard spatially conservative scheme, these physically conserved quantities are naturally conserved numerically. This is not the case if one uses the microscopic 
equation for the particle density distribution $f$ and then takes moments from the discrete $f$, since many collision solvers, for example the spectral methods [8, 31, 9, 30, do not have the exact conservation properties, and extra efforts are needed for the exact conservation, see [27, 34, 7]. The advantage of the conservation of moments made from the macro system was noted and emphasized in [15.

Numerically conserving the physically conserved quantities is a highly desirable property for a numerical scheme. In this paper we realized this using the moment systems which are already in conservation forms at the continuous level. Note that although not all AP schemes use the moment system, some of the popular ones, like those in Filbet-Jin [6], JinYan [15] and micro-macro decomposition based method 2, indeed use it thus naturally induce the exactly conservative schemes. This fact was pointed out and utilized in [15. In Section 7 we further extend this idea to design conservative schemes for general (collisional or non-collisional) kinetic systems, using the Vlasov-Poisson and Vlasov-Poisson-Boltzmann systems as examples. The general principle favored here is that one should solve the original kinetic equation and the moment system simultaneously. One first obtains the moment system analytically and then the discrete moment system, when using spatially conservative discretizations, automatically yields the exact conservations of moments, if they are conserved physically. Since the total energy also includes the electric energy, another idea introduced here is to replace the Poisson equation for the electric field by the Ampère equation, and then the coupled system is discretized in time by a carefully designed explicit-implicit scheme.

This paper is organized as follows. Section 2 gives an introduction of two kinetic equations: the Boltzmann and the Fokker-Planck-Landau equations. In Section 3 the basic idea of the micro-macro decomposition method is reviewed. Section 4 studies the fully discretized AP numerical scheme, especially on how to embed the penalization method in the micro-macro decomposition framework to solve the full nonlinear Boltzmann and Fokker-Planck-Landau equations. We also emphasize that our scheme conserves the moments (mass, momentum, energy) if these moment variables are obtained from the macroscopic system instead of from the particle density distribution $f$. Section 5 provides some implementation details, while in Section 6] number of numerical examples are used to study the conservation property as well as the performance of the new schemes in different regimes. In Section 7 we introduce conservation schemes for the Vlasov-Ampére system and Vlasov-Ampre-Boltzmann system, with the conservations obtained through solving the moment systems and a specially designed time discretization. Finally, we conclude and list some future work in Section 8

\section{Introduction of two kinetic equations}

\subsection{The Boltzmann equation}

One of the most celebrated kinetic equations for rarefied gas is the Boltzmann equation, which describes the time evolution of the density distribution of a dilute gas of particles when the only interactions considered are binary elastic collisions. A dimensionless form reads

$$
\partial_{t} f+v \cdot \nabla_{x} f=\frac{1}{\varepsilon} \mathcal{Q}_{\mathrm{B}}(f, f), \quad t>0,(x, v) \in \Omega \times \mathbb{R}^{d},
$$

where $f(t, x, v)$ is the probability density distribution (p.d.f) function, modeling the probability of finding a particle at time $t$, at position $x \in \Omega$, with velocity $v \in \mathbb{R}^{d}$. The parameter $\varepsilon$ is the Knudsen number defined as the ratio of the mean free path over a typical length scale such as the size of the spatial domain, which characterizes the degree of rarefaction of 
the gas. The Boltzmann collision operator is denoted by $\mathcal{Q}_{\mathrm{B}}$, which is a bilinear functional and only acts on the velocity dependence of $f$,

$\mathcal{Q}_{B}(f, g)(t, x, v)=\int_{\mathbb{R}^{d}} \int_{\mathbb{S}^{d-1}} B\left(\left|v-v_{*}\right|, \cos \theta\right)\left(f\left(t, x, v^{\prime}\right) g\left(t, x, v_{*}^{\prime}\right)-f(t, x, v) g\left(t, x, v_{*}\right)\right) d \sigma d v_{*}$.

We consider the elastic interaction. The velocity pairs before and after the collision $\left(v, v_{*}\right)$ and $\left(v^{\prime}, v_{*}^{\prime}\right)$ have the relation,

$$
\left\{\begin{array}{l}
v^{\prime}=\frac{v+v_{*}}{2}+\frac{\left|v-v_{*}\right|}{2} \sigma \\
v_{*}^{\prime}=\frac{v+v_{*}}{2}-\frac{\left|v-v_{*}\right|}{2} \sigma
\end{array}\right.
$$

Here $\sigma$ is the scattering direction varying in the unit sphere $\mathbb{S}^{d-1}$, and is defined by

$$
\sigma=\frac{u^{\prime}}{\left|u^{\prime}\right|}=\frac{u^{\prime}}{|u|}
$$

where the pre- and post-collisional relative velocities $u=v-v_{*}$ and $u^{\prime}=v^{\prime}-v_{*}^{\prime}$ have the same magnitude, i.e., $\left|u^{\prime}\right|=|u|$.

Cosine of the deviation angle is given by

$$
\cos \theta=\frac{u \cdot u^{\prime}}{|u|^{2}}=\frac{u \cdot \sigma}{|u|}:=\hat{u} \cdot \sigma .
$$

The collision kernel $B$ is a non-negative function, which is usually written in a form of a product of a power function of the relative velocity $u$ and a scattering angular function $b$ depending on $\cos \theta$, that is,

$$
B\left(\left|v-v_{*}\right|, \cos \theta\right)=B(|u|, \hat{u} \cdot \sigma)=C_{\lambda}|u|^{\lambda} b(\hat{u} \cdot \sigma), \quad-d \leq \lambda \leq 1 .
$$

Here $\lambda>0$ corresponds to the hard potentials, $\lambda<0$ the soft potentials, and $\lambda=0$ refers to the Maxwell pseudo-molecules model.

It is not hard to find that

$$
\int_{\mathbb{R}^{d}} \mathcal{Q}_{B}(f, f)(v) \phi(v) d v=\frac{1}{2} \int_{\mathbb{R}^{d}} f f_{*}\left(\phi+\phi_{*}-\phi^{\prime}-\phi_{*}^{\prime}\right) B\left(\left|v-v_{*}\right|, \cos \theta\right) d \sigma d v_{*}
$$

equals to zeros if

$$
\phi+\phi_{*}=\phi^{\prime}+\phi_{*}^{\prime} .
$$

One can prove that 2.6 holds if and only if $\phi(v)$ lies in the space spanned by the moments of mass, momentum and kinetic energy. We call the $d+2$ test functions $1, v, \frac{|v|^{2}}{2}$ collision invariants associated to $\mathcal{Q}_{B}$. Denote

$$
m(v)=\left(1, v, \frac{|v|^{2}}{2}\right)^{T}
$$

then

$$
\int_{\mathbb{R}^{d}} \mathcal{Q}_{B}(f, f) m(v) d v=0
$$

which correspond to the conservation of mass, momentum and kinetic energy of $\mathcal{Q}_{B}$.

Define $U=(\rho, \rho u, E)^{T}$ as the velocity averages of $f$ multiplying by the collision invariants $m$, which is a vector composing of $d+2$ conserved moments of density, momentum and energy,

$$
\langle m M(U)\rangle=U=\int_{\mathbb{R}^{d}}\left(\begin{array}{c}
1 \\
v \\
\frac{1}{2}|v|^{2}
\end{array}\right) f(v) d v=\left(\begin{array}{c}
\rho \\
\rho u \\
\frac{1}{2} \rho|u|^{2}+\frac{d}{2} \rho T
\end{array}\right)=\left(\begin{array}{c}
\rho \\
\rho u \\
E
\end{array}\right) .
$$


If setting $\phi(v)=\ln f(v)$ in 2.5 , one can prove the following dissipation of entropy

$$
\int_{\mathbb{R}^{d}} \mathcal{Q}_{B}(f, f) \ln f d v \leq 0,
$$

which is known as the celebrated Boltzmann's H-theorem. Furthermore, the Boltzmann theorem for elastic interaction is given by

$$
\int_{\mathbb{R}^{d}} \mathcal{Q}_{B}(f, f) \ln f d v=0 \Leftrightarrow \mathcal{Q}_{B}(f, f)=0 \Leftrightarrow f=M,
$$

where $M$ is the equilibrium state given by a Maxwellian distribution

$$
M(U)(v)=\frac{\rho}{(2 \pi T)^{\frac{d}{2}}} \exp \left(-\frac{|v-u|^{2}}{2 T}\right):=M_{U(x, t)}(v) .
$$

Here $\rho, u$ and $T$ are respectively the density, bulk velocity, and temperature defined by

$$
\rho=\int_{\mathbb{R}^{d}} f(v) d v, \quad u=\frac{1}{\rho} \int_{\mathbb{R}^{d}} f(v) v d v, \quad T=\frac{1}{d \rho} \int_{\mathbb{R}^{d}} f(v)|v-u|^{2} d v .
$$

The fluid limit We introduce the notation $\langle\cdot\rangle$ as the velocity averages of the argument, i.e.,

$$
\langle f\rangle=\int_{\mathbb{R}^{d}} f(v) d v .
$$

Multiplying 2.1 by $m(v)$ and integrating with respect to $v$, by using the conservation property of $\mathcal{Q}_{\mathrm{B}}$ given by 2.7 , one has

$$
\partial_{t}\langle m f\rangle+\nabla_{x} \cdot\langle v m f\rangle=0
$$

This gives a non-closed system of conservation laws

$$
\partial_{t}\left(\begin{array}{c}
\rho \\
\rho u \\
E
\end{array}\right)+\nabla_{x} \cdot\left(\begin{array}{c}
\rho u \\
\rho u \otimes u+\mathbb{P} \\
E u+\mathbb{P} u+\mathbb{Q}
\end{array}\right)=0
$$

where $E$ is the energy defined in 2.8$), \mathbb{P}=\langle(v-u) \otimes(v-u) f\rangle$ is the pressure tensor, and $\mathbb{Q}=\frac{1}{2}\left\langle(v-u)|v-u|^{2} f\right\rangle$ is the heat flux vector. When $\varepsilon \rightarrow 0, f \rightarrow M(U)$. Replacing $f$ by $M(U)$ and using expression $2.11, \mathbb{P}$ and $\mathbb{Q}$ are given by

$$
\mathbb{P}=p I, \quad \mathbb{Q}=0,
$$

where $p=\rho T$ is the pressure, $I$ is the identity matrix. Then 2.12 reduces to the usual compressible Euler equations

$$
\partial_{t}\left(\begin{array}{c}
\rho \\
\rho u \\
E
\end{array}\right)+\nabla_{x} \cdot\left(\begin{array}{c}
\rho u \\
\rho u \otimes u+p I \\
(E+p) u
\end{array}\right)=0 .
$$

\subsection{The Fokker-Planck-Landau equation}

The nonlinear Fokker-Planck-Landau (nFPL) equation is widely used in plasma physics. The rescaled nFPL equation reads

$$
\partial_{t} f+v \cdot \nabla_{x} f=\frac{1}{\varepsilon} \mathcal{Q}_{\mathrm{L}}(f, f), \quad t>0,(x, v) \in \Omega \times \mathbb{R}^{d},
$$


with the nFPL operator

$$
\mathcal{Q}_{L}(f, f)=\nabla_{v} \cdot \int_{\mathbb{R}^{d}} A\left(v-v_{*}\right)\left(f\left(v_{*}\right) \nabla_{v} f(v)-f(v) \nabla_{v} f\left(v_{*}\right)\right) d v_{*},
$$

where the semi-positive definite matrix $A(z)$ is

$$
A(z)=\Psi(z)\left(I-\frac{z \otimes z}{|z|^{2}}\right), \quad \Psi(z)=|z|^{\gamma+2} .
$$

The parameter $\gamma$ characterizes the type of interaction between particles. The inverse power law gives $\gamma \geq-3$. Similar to Boltzmann collision operator, $\gamma>0$ categorizes hard potentials, $\gamma=0$ for Maxwellian molecules and $\gamma<0$ for soft potentials. The case $\gamma=-3$ corresponding to Coulomb interactions.

The nFPL equation is derived as a limit of the Boltzmann equation when all the collisions become grazing. Therefore, the nFPL operator possesses similar conservation laws and decay of entropy (H-theorem) as the Boltzmann collision operator, which are given in $2.97-(2.10)$.

\section{The micro-macro decomposition method}

When no confusion is possible, we set $M_{U(x, t)}(v)=M$ in the following. Consider the Hilbert space $L_{M}^{2}=\left\{\phi \mid \phi M^{-\frac{1}{2}} \in L^{2}\left(\mathbb{R}^{d}\right)\right\}$ endowed with the weighted scalar product

$$
(\phi, \psi)_{M}=\left\langle\phi \psi M^{-1}\right\rangle \text {. }
$$

It is well-known that the linearized operator $\mathcal{L}_{M}$ is a non-positive self-adjoint operator on $L_{M}^{2}$ and that its null space is

$$
\mathcal{N}\left(\mathcal{L}_{M}\right)=\operatorname{Span}\left\{M,|v| M,|v|^{2} M\right\}
$$

whose orthogonal basis is

$$
\mathcal{B}=\left\{\frac{M}{\rho}, \frac{(v-u)}{\sqrt{T}} \frac{M}{\rho},\left(\frac{|v-u|^{2}}{2 T}-\frac{d}{2}\right) \frac{M}{\rho}\right\} .
$$

The orthogonal projection of $\phi \in L_{M}^{2}$ onto $\mathcal{N}\left(\mathcal{L}_{M}\right)$ is given by $\Pi_{M}(\phi)$ :

$$
\Pi_{M}(\phi)=\frac{1}{\rho}\left[\langle\phi\rangle+\frac{(v-u) \cdot\langle(v-u) \phi\rangle}{T}+\left(\frac{|v-u|^{2}}{2 T}-\frac{d}{2}\right) \frac{2}{d}\left\langle\left(\frac{|v-u|^{2}}{2 T}-\frac{d}{2}\right) \phi\right\rangle\right] M .
$$

We explain the main idea of the micro-macro decomposition, which mostly follows that in [2], where the BGK equation, with $\mathcal{Q}_{B G K}(f, f)=\frac{1}{\tau}(M-f)$, is numerically implemented ( $\tau$ is the relaxation time). Let $f$ be the solution of the Boltzmann equation (2.1). We decompose $f=f(t, x, v)$ as

$$
f=M+\varepsilon g(x, t, v)
$$

where $U$ and $M$ are given in 2.8 and 2.11 respectively. Inserting (3.1) into 2.1), one obtains

$$
\partial_{t} M+v \cdot \nabla_{x} M+\varepsilon\left(\partial_{t} g+v \cdot \nabla_{x} g\right)=\frac{1}{\varepsilon} \mathcal{Q}(M+\varepsilon g, M+\varepsilon g) .
$$

Denote the linearized collision operator

$$
\mathcal{L}_{M}(g)=2 \mathcal{Q}(M, g)
$$

Since $\mathcal{Q}$ is bilinear and $\mathcal{Q}(M, M)=0$, then

$$
\mathcal{Q}(M+\varepsilon g, M+\varepsilon g)=\mathcal{Q}(M, M)+2 \varepsilon \mathcal{Q}(M, g)+\varepsilon^{2} \mathcal{Q}(g, g)=\varepsilon \mathcal{L}_{M}(g)+\varepsilon^{2} \mathcal{Q}(g, g),
$$


thus

$$
\partial_{t} M+v \cdot \nabla_{x} M+\varepsilon\left(\partial_{t} g+v \cdot \nabla_{x} g\right)=\mathcal{L}_{M}(g)+\varepsilon \mathcal{Q}(g, g) .
$$

Applying the operator $\mathbb{I}-\Pi_{M}$ to $(3.3)$, one gets

$$
\partial_{t} g+\left(\mathbb{I}-\Pi_{M}\right)\left(v \cdot \nabla_{x} g\right)-\mathcal{Q}(g, g)=\frac{1}{\varepsilon}\left[\mathcal{L}_{M}(g)-\left(\mathbb{I}-\Pi_{M}\right)\left(v \cdot \nabla_{x} M\right)\right] .
$$

On the other hand, if we take the moments of equation (3.3), then

$$
\partial_{t}\langle m M\rangle+\nabla_{x} \cdot\langle v m M\rangle+\varepsilon \nabla_{x} \cdot\langle v m g\rangle=0 .
$$

Denote the flux vector of $U$ by

$$
F(U)=\langle v m M\rangle=\left(\begin{array}{c}
\rho u \\
\rho u \otimes u+\rho T \\
E u+\rho T u
\end{array}\right),
$$

then 3.5 becomes

$$
\partial_{t} U+\nabla_{x} \cdot F(U)+\varepsilon \nabla_{x} \cdot\langle v m g\rangle=0 .
$$

Therefore, the coupled system $(3.4)$ and $(3.6)$ gives a kinetic/fluid formulation of the Boltzmann equation. It has been shown in [2] that this coupled system is equivalent to the Boltzmann equation 2.1.

\section{Initial and boundary conditions}

For the initial condition, we set

$$
f(t=0, x, v)=f^{0}(x, v) .
$$

$x$ is in a bounded set $\Omega$ with boundary $\Gamma$. For the numerical implementation purpose, we only consider the periodic boundary condition (BC) in $x$ in this paper. Nevertheless, we briefly mention other types of BC.

For points $x$ on the boundary $\Gamma$, the distribution function of incoming velocities (i.e., $v$ with $v \cdot n(x)<0$, where $n(x)$ is the outer normal vector of $\Gamma$ at $x$ ) should be specified. The Dirichlet $\mathrm{BC}$ reads

$$
f(t, x, v)=f_{\Gamma}(t, x, v) \quad \forall x \in \Gamma, \forall v \text {, s.t. } v \cdot n(x)<0 .
$$

The reflecting $\mathrm{BC}$ is given by

$$
f(t, x, v)=\int_{v^{\prime} \cdot n(x)>0} K\left(x, v, v^{\prime}\right) f\left(t, x, v^{\prime}\right) d v^{\prime} \quad \forall x \in \Gamma, \forall v, \text { s.t. } v \cdot n(x)<0,
$$

where the kernel $K$ satisfies the zero normal mass flux condition across the boundary:

$$
\int_{\Gamma} v \cdot n(x) f(t, x, v) d v=0
$$

The periodic BC can be used when the shape of $\Omega$ is symmetric,

$$
f(t, x, v)=f(t, S x, v), \quad x \in \Gamma_{1}, \forall v,
$$

where $S$ is a one-to-one mapping from a part $\Gamma_{1}$ of $\Gamma$ onto another part $\Gamma_{2}$ of $\Gamma$.

In general, using the micro-macro decomposition into boundary conditions (3.7)-3.8 provides relations for $M+\varepsilon g$, but do not provide the values for $M$ and $g$ separately. Moreover, $f$ is generally known only for incoming velocities at boundary points, which may induce difficulties to define the macroscopic moments $U$. Note that various numerical boundary conditions based on micro-macro formulation for linear kinetic equations in the diffusion limit is studied in [19]. 


\section{Numerical Approximation}

\subsection{Time discretization}

We denote $\Delta t$ a fixed time step, $t_{n}$ a discrete time with $t_{n}=n \Delta t, n \in \mathbb{N}$. Let $U^{n}(x) \approx$ $U\left(t_{n}, x\right), g^{n}(x, v) \approx g\left(t_{n}, x, v\right)$. Note that in equation $3.4, \varepsilon^{-1} \mathcal{L}_{M}(g)$ is the only collision term that presents the stiffness, thus one needs to take an implicit discretization for this term, while the term $\left(I-\Pi_{M}\right)\left(v \cdot \nabla_{x} M\right)$ is still explicit. The time discretization for (3.4) is given by

$$
\frac{g^{n+1}-g^{n}}{\Delta t}+\left(\mathbb{I}-\Pi_{M^{n}}\right)\left(v \cdot \nabla_{x} g^{n}\right)-\mathcal{Q}\left(g^{n}, g^{n}\right)=\frac{1}{\varepsilon}\left[\mathcal{L}_{M^{n}}\left(g^{n+1}\right)-\left(\mathbb{I}-\Pi_{M^{n}}\right)\left(v \cdot \nabla_{x} M^{n}\right)\right] .
$$

For the time discretization of the fluid part (3.6), the flux $F(U)$ at time $t_{n}$ is approximated by $F\left(U^{n}\right)=\left\langle v m M^{n}\right\rangle$, and the convection term $\nabla_{x} \cdot\langle v m g\rangle$ is discretized by $\nabla_{x} \cdot\left\langle v m g^{n+1}\right\rangle$,

$$
\frac{U^{n+1}-U^{n}}{\Delta t}+\nabla_{x} \cdot F\left(U^{n}\right)+\varepsilon \nabla_{x} \cdot\left\langle v m g^{n+1}\right\rangle=0 .
$$

In [2] only BGK collision operator was considered, thus avoided the difficulty of inverting the $\mathcal{L}_{M^{n}}\left(g^{n+1}\right)$ term in 4.1 , since the implicit BGK operator can be inverted explicitly, thanks to the conservation property of the operator due to 2.8. For general collision operator this is no longer true. In the next subsection, we propose an efficient method to deal with the term $\mathcal{L}_{M^{n}}\left(g^{n+1}\right)$, which is one of the main ideas of this paper.

\subsection{AP schemes by penalization}

To avoid the complication of inverting the stiff, implicit linearized collision operator $\mathcal{L}_{M^{n}}\left(g^{n+1}\right)$ in 4.1 , our proposed method is to use the relation

$$
\mathcal{Q}(M, g)=\frac{1}{4}[\mathcal{Q}(M+g, M+g)-\mathcal{Q}(M-g, M-g)]
$$

and by 3.2 , namely $\mathcal{L}_{M}(g)=2 \mathcal{Q}(M, g)$, then

$$
\mathcal{L}_{M^{n}}\left(g^{n+1}\right)=\frac{1}{2}\left[\mathcal{Q}\left(M^{n}+g^{n+1}, M^{n}+g^{n+1}\right)-\mathcal{Q}\left(M^{n}-g^{n+1}, M^{n}-g^{n+1}\right)\right] .
$$

To deal with the implicit collision operator $\mathcal{Q}$, we adopt the penalization method developed in [6] for the Boltzmann equation, and that in [15] for the Fokker-Planck-Landau equation.

We briefly recall the spirit of the penalization for the collision operators used in [6, 15]. They introduced some dissipative penalization operator $\mathcal{P}$ for the Boltzmann or the FPL collision operator. The collision operators $\mathcal{Q}_{B}$ and $\mathcal{Q}_{L}$ in 2.1 or 2.14, when divided by a small Knudsen number $\varepsilon$, become numerically stiff. Since explicit schemes require severe stability constraints and are computationally expensive, while implicit schemes, though allow larger time step, are difficult to seek numerical solution of a fully nonlinear problem at each time step, thus one desires to combine both advantages of implicit and explicit schemes for solving the stiff problem: large time step and low computational complexity.

The idea is to split the RHS of (2.1) or 2.14 as the sum of a stiff part and a less stiff part as

$$
\frac{\mathcal{Q}(f, f)}{\varepsilon}=\underbrace{\frac{\mathcal{Q}\left(f^{n}, f^{n}\right)-\mathcal{P}\left(f^{n}\right)}{\varepsilon}}_{\text {less stiff }}+\underbrace{\frac{\mathcal{P}\left(f^{n+1}\right)}{\varepsilon}}_{\text {stiff }},
$$


where $\mathcal{Q}$ represents $\mathcal{Q}_{B}$ or $\mathcal{Q}_{L}, \mathcal{P}(f)$ is a well balanced, linear operator and is asymptotically close to the source term $\mathcal{Q}(f, f)$. We adopt a first order implicit-explicit (IMEX) scheme for the time discretization here.

The following gives an explicit explanation on how the linearized operator is implemented by combining the formulation (4.3) and the penalization strategies for the Boltzmann and the FPL equations. The advantage of using BGK for the Boltzmann and linear FokkerPlanck for the FPL equation is that these penalty operators are much easier to invert than the original kinetic operators when discretized implicitly. In particular, the implicit BGK operator can be inverted explicitly, while the Fokker-Planck operator can be inverted as a linear symmetric operator. See [6, 15].

I. For the Boltzmann equation, the linear BGK collision operator [6]

$$
P(f)=P_{B G K}^{M} f=\beta(M-f)
$$

is used as the penalty operator. Now we replace $\mathcal{L}_{M^{n}}\left(g^{n+1}\right)$ in 4.2 by $\mathcal{L}_{M^{n}}^{P}\left(g^{n+1}\right)$, given by

$$
\begin{aligned}
\mathcal{L}_{M^{n}}^{P}\left(g^{n+1}\right)= & \frac{1}{2}\left[\mathcal{Q}_{\mathrm{B}}\left(M^{n}+g^{n}, M^{n}+g^{n}\right)-\beta_{1}^{n}\left(M^{n}-\left(M^{n}+g^{n}\right)\right)+\beta_{1}^{n+1}\left(M^{n+1}-\left(M^{n+1}+g^{n+1}\right)\right)\right. \\
& \left.-\left\{\mathcal{Q}_{\mathrm{B}}\left(M^{n}-g^{n}, M^{n}-g^{n}\right)-\beta_{2}^{n}\left(M^{n}-\left(M^{n}-g^{n}\right)\right)+\beta_{2}^{n+1}\left(M^{n+1}-\left(M^{n+1}-g^{n+1}\right)\right)\right\}\right] \\
= & \frac{1}{2}\left[\mathcal{Q}_{\mathrm{B}}\left(M^{n}+g^{n}, M^{n}+g^{n}\right)+\beta_{1}^{n} g^{n}-\beta_{1}^{n+1} g^{n+1}\right. \\
& \left.\left.-\mathcal{Q}_{\mathrm{B}}\left(M^{n}-g^{n}, M^{n}-g^{n}\right)+\beta_{2}^{n} g^{n}-\beta_{2}^{n+1} g^{n+1}\right)\right] \\
= & \frac{1}{2}\left[\mathcal{Q}_{\mathrm{B}}\left(M^{n}+g^{n}, M^{n}+g^{n}\right)-\mathcal{Q}_{\mathrm{B}}\left(M^{n}-g^{n}, M^{n}-g^{n}\right)\right] \\
& +\frac{1}{2}\left(\beta_{1}^{n}+\beta_{2}^{n}\right) g^{n}-\frac{1}{2}\left(\beta_{1}^{n+1}+\beta_{2}^{n+1}\right) g^{n+1} .
\end{aligned}
$$

In the Boltzmann equation, the parameter $\beta>0$ is chosen as an upper bound of $\|\nabla \mathcal{Q}(M)\|$ or some approximation of it, for example,

$$
\begin{aligned}
& \beta_{1}^{n}=\sup _{v}\left|\frac{\mathcal{Q}\left(M^{n}+g^{n}, M^{n}+g^{n}\right)-\mathcal{Q}\left(M^{n}, M^{n}\right)}{g^{n}}\right|=\sup _{v}\left|\frac{\mathcal{Q}\left(M^{n}+g^{n}, M^{n}+g^{n}\right)}{g^{n}}\right|, \\
& \beta_{2}^{n}=\sup _{v}\left|\frac{\mathcal{Q}\left(M^{n}-g^{n}, M^{n}-g^{n}\right)-\mathcal{Q}\left(M^{n}, M^{n}\right)}{g^{n}}\right|=\sup _{v}\left|\frac{\mathcal{Q}\left(M^{n}-g^{n}, M^{n}-g^{n}\right)}{g^{n}}\right| .
\end{aligned}
$$

II. For the nFPL equation, the linear Fokker-Planck (FP) operator

$$
P(f)=P_{F P}^{M} f=\nabla_{v} \cdot\left(M \nabla_{v}\left(\frac{f}{M}\right)\right)
$$

is chosen as the suitable penalty operator [15]. We now replace $\mathcal{L}_{M^{n}}\left(g^{n+1}\right)$ in 4.2 by 
$\mathcal{L}_{M^{n}}^{P}\left(g^{n+1}\right)$ (and use the bracket notation $(\cdot)$ to denote $P$ imposed on the argument),

$$
\begin{aligned}
\mathcal{L}_{M^{n}}^{P}\left(g^{n+1}\right)= & \frac{1}{2}\left[\mathcal{Q}_{\mathrm{L}}\left(M^{n}+g^{n}, M^{n}+g^{n}\right)-\beta_{1}^{n} P^{n}\left(M^{n}+g^{n}\right)+\beta_{1}^{n} P^{n+1}\left(M^{n+1}+g^{n+1}\right)\right. \\
& \left.-\left\{\mathcal{Q}_{\mathrm{L}}\left(M^{n}-g^{n}, M^{n}-g^{n}\right)-\beta_{2}^{n} P^{n}\left(M^{n}-g^{n}\right)+\beta_{2}^{n} P^{n+1}\left(M^{n+1}-g^{n+1}\right)\right\}\right] \\
= & \frac{1}{2}\left[\mathcal{Q}_{\mathrm{L}}\left(M^{n}+g^{n}, M^{n}+g^{n}\right)-\beta_{1}^{n} P^{n}\left(g^{n}\right)+\beta_{1}^{n} P^{n+1}\left(g^{n+1}\right)\right. \\
& \left.-\mathcal{Q}_{\mathrm{L}}\left(M^{n}-g^{n}, M^{n}-g^{n}\right)-\beta_{2}^{n} P^{n}\left(g^{n}\right)+\beta_{2}^{n} P^{n+1}\left(g^{n+1}\right)\right] \\
= & \frac{1}{2}\left[\mathcal{Q}_{\mathrm{L}}\left(M^{n}+g^{n}, M^{n}+g^{n}\right)-\mathcal{Q}_{\mathrm{L}}\left(M^{n}-g^{n}, M^{n}-g^{n}\right)\right] \\
& -\frac{1}{2}\left(\beta_{1}^{n}+\beta_{2}^{n}\right) P^{n}\left(g^{n}\right)+\frac{1}{2}\left(\beta_{1}^{n}+\beta_{2}^{n}\right) P^{n+1}\left(g^{n+1}\right),
\end{aligned}
$$

where the well-balanced property of $P$, i.e., $P^{n}\left(M^{n}\right)=P^{n+1}\left(M^{n+1}\right)=0$ is used.

In $4.8, \beta_{1}^{n}$ and $\beta_{2}^{n}$ are chosen as

$$
\begin{aligned}
& \beta_{1}^{n}=\beta_{0} \max _{v} \lambda\left(D_{A}\left(g^{n}+M^{n}\right)\right), \\
& \beta_{2}^{n}=\beta_{0} \max _{v} \lambda\left(D_{A}\left(g^{n}-M^{n}\right)\right) .
\end{aligned}
$$

$\beta_{0}$ is a constant satisfying $\beta_{0}>\frac{1}{2}$, and a simple choice is $\beta_{0}=1 . \lambda\left(D_{A}\right)$ is the spectral radius of the positive symmetric matrix $D_{A}$,

$$
D_{A}(f)=\int_{\mathbb{R}^{d}} A\left(v-v_{*}\right) f_{*} d v_{*}
$$

\subsection{Space and velocity discretizations}

Space discretization For simplicity and clarity of notations, we only consider $x \in \mathbb{R}$. As done in [2], a finite volume discretization is used for the transport term in the left-handside of 4.1); a central difference scheme is used to discretize the term $\left(\mathbb{I}-\Pi_{M^{n}}\right)\left(v \cdot \nabla_{x} M^{n}\right)$ via 4.1, and the term $\varepsilon \nabla_{x} \cdot\left\langle v m g^{n+1}\right\rangle$ via 4.2 .

Consider spatial grid points $x_{i+\frac{1}{2}}$ and $x_{i}$ the center of the cell $\left[x_{i-\frac{1}{2}}, x_{i+\frac{1}{2}}\right]$, for $i=$ $0, \cdots N_{x}$. A uniform space step is $\Delta x=x_{i+\frac{1}{2}}-x_{i-\frac{1}{2}}=x_{i}-x_{i-1}$. Let $U_{i}^{n} \approx U\left(t_{n}, x_{i}\right)$ and $g_{i+\frac{1}{2}}^{n} \approx g\left(t_{n}, x_{i+\frac{1}{2}}\right)$. Now we define the following notations for the finite difference operators. For every grid function $\phi=\left(\phi_{i+\frac{1}{2}}\right)$, define the one-sided difference operators:

$$
D^{-} \phi_{i+\frac{1}{2}}=\frac{\phi_{i+\frac{1}{2}}-\phi_{i-\frac{1}{2}}}{\Delta x}, \quad D^{+} \phi_{i+\frac{1}{2}}=\frac{\phi_{i+\frac{3}{2}}-\phi_{i+\frac{1}{2}}}{\Delta x} .
$$

For every grid function $\mu=\left(\mu_{i}\right)$, we define the following centered operator:

$$
\delta^{0} \mu_{i+\frac{1}{2}}=\frac{\mu_{i+1}-\mu_{i}}{\Delta x} .
$$

Velocity discretization We adopt the simple trapezoidal rule to compute the numerical integral in velocity space. For example, we write the one-dimensional trapezoidal rule,

$$
\int_{\mathbb{R}} f d v \approx \Delta v\left(\frac{1}{2} f\left(v_{0}\right)+f\left(v_{1}\right)+\cdots+f\left(v_{N_{v}-1}\right)+\frac{1}{2} f\left(v_{N_{v}}\right)\right):=\sum_{j=0}^{N_{v}} f\left(v_{j}\right) w_{j} \Delta v
$$

where $w=\left(\frac{1}{2}, 1, \cdots, 1, \frac{1}{2}\right)$. 


\section{Macroscopic equations}

The fluid equation (4.2) is approximated at points $x_{i}$. The flux $\partial_{x} F\left(U^{n}\right)$ at $x_{i}$ is discretized by

$$
\left.\partial_{x} F\left(U^{n}\right)\right|_{x_{i}} \approx \frac{F_{i+\frac{1}{2}}\left(U^{n}\right)-F_{i-\frac{1}{2}}\left(U^{n}\right)}{\Delta x}
$$

where upwind-based discretization is used to approximate $F\left(U^{n}\right)=\left\langle v m M^{n}\right\rangle$ at points $x_{i+\frac{1}{2}}$. The first order approximation is given by

$$
F_{i+\frac{1}{2}}\left(U^{n}\right)=\left\langle m\left(v^{+} M_{i}^{n}+v^{-} M_{i+1}^{n}\right)\right\rangle .
$$

A second order approximation of $\partial_{x} F(U)$ term will be discussed in section 5

The flux term $\partial_{x}\left\langle v m g^{n+1}\right\rangle$ at $x_{i}$ on the right-hand-side of 4.2 is approximated by central differences,

$$
\left.\partial_{x}\left\langle v m g^{n+1}\right\rangle\right|_{x_{i}} \approx\left\langle v m \frac{g_{i+\frac{1}{2}}^{n+1}-g_{i-\frac{1}{2}}^{n+1}}{\Delta x}\right\rangle
$$

The fully discretized scheme of the equation 4.2 then reads

$$
\frac{U_{i}^{n+1}-U_{i}^{n}}{\Delta t}+\frac{F_{i+\frac{1}{2}}\left(U^{n}\right)-F_{i-\frac{1}{2}}\left(U^{n}\right)}{\Delta x}=-\varepsilon \sum_{j=0}^{N_{v}} v_{j} m\left(v_{j}\right) \frac{g_{i+\frac{1}{2}, j}^{n+1}-g_{i-\frac{1}{2}, j}^{n+1}}{\Delta x} w_{j} \Delta v
$$

where $g_{i+\frac{1}{2}, j}^{n} \approx g\left(t_{n}, x_{i+\frac{1}{2}}, v_{j}\right)$.

Next, we prove that the discrete macroscopic equations 4.12 conserve mass, momentum and total energy.

Theorem 4.1. (Conservation of moments $U$ )

For periodic or zero flux boundary condition, one has

$$
\sum_{i=0}^{N_{x}} U_{i}^{n+1}=\sum_{i=0}^{N_{x}} U_{i}^{n}
$$

Namely, the total mass, momentum and energy are all numerically conserved.

Proof. Summing up all $i=0, \cdots, N_{x}$ on 4.12 , one has

$$
\frac{\sum_{i} U_{i}^{n+1}-\sum_{i} U_{i}^{n}}{\Delta t}+\sum_{i}\left(\frac{F_{i+\frac{1}{2}}\left(U^{n}\right)-F_{i-\frac{1}{2}}\left(U^{n}\right)}{\Delta x}\right)=-\varepsilon \sum_{i} \sum_{j=0}^{N_{v}} v_{j} m\left(v_{j}\right) \frac{g_{i+\frac{1}{2}, j}^{n+1}-g_{i-\frac{1}{2}, j}^{n+1}}{\Delta x} w_{j} \Delta v .
$$

By the assumption on the boundary condition, the telescoping summation terms vanish, and then one has 4.13.

Remark 4.2. If $\varepsilon$ is spatially dependent, then (4.2) is written by

$$
\frac{U^{n+1}-U^{n}}{\Delta t}+\nabla_{x} \cdot F\left(U^{n}\right)+\nabla_{x} \cdot\left\langle\varepsilon v m g^{n+1}\right\rangle=0
$$

and 4.14) correspondingly becomes

$$
\begin{aligned}
& \frac{\sum_{i} U_{i}^{n+1}-\sum_{i} U_{i}^{n}}{\Delta t}+\sum_{i}\left(\frac{F_{i+\frac{1}{2}}\left(U^{n}\right)-F_{i-\frac{1}{2}}\left(U^{n}\right)}{\Delta x}\right) \\
= & -\sum_{i} \sum_{j=0}^{N_{v}} v_{j} m\left(v_{j}\right) \frac{\varepsilon_{i+\frac{1}{2}} g_{i+\frac{1}{2}, j}^{n+1}-\varepsilon_{i-\frac{1}{2}} g_{i-\frac{1}{2}, j}^{n+1}}{\Delta x} w_{j} \Delta v,
\end{aligned}
$$

with $\varepsilon_{i+\frac{1}{2}}=\varepsilon\left(x_{i+\frac{1}{2}}\right), \varepsilon_{i-\frac{1}{2}}=\varepsilon\left(x_{i-\frac{1}{2}}\right)$. This again has the conservation property 4.13). 
Remark 4.3. Typically, a discrete collision operator, particularly those based on spectral approximations in velocity space [8, [31, 9, 30], does not conserve exactly the moments $U$ (4.13), which needs to be taken care of with extra efforts [27, 34, 7]. What differs here is that the conserved variables $U$ are obtained from the macroscopic system (3.6), which has the zero right hand side, thus the conservation property (4.13) can be easily guaranteed by any conservative discretization of the spatial derivative in (3.6). What differs here from those typical kinetic solvers in [34, 2] is that in the latter cases the moments were obtained by taking the discrete moments from $f$, computed from the original kinetic equation for $f$, with the collision operator discretized not in an exactly conserved way! This observation is not new, and in fact was already pointed out in [15]. In section 7 this point will be further explored for general kinetic systems and this offers a generic recipe for obtaining (exactly) conservative schemes through solving the moment systems.

\section{Microscopic equation}

Equation 4.1 is approximated at grid point $x_{i+\frac{1}{2}}$; the term $\left(\mathbb{I}-\Pi_{M^{n}}\right)\left(v \cdot \nabla_{x} g^{n}\right)$ in the left-hand-side is approximated by a first order upwind scheme

$$
\left.\left(\mathbb{I}-\Pi_{M^{n}}\right)\left(v \partial_{x} g^{n}\right)\right|_{x_{i+\frac{1}{2}}} \approx\left(\mathbb{I}-\Pi_{i+\frac{1}{2}}^{n}\right)\left(v^{+} D^{-}+v^{-} D^{+}\right) g_{i+\frac{1}{2}}^{n} \cdot
$$

The transport term $\left(\mathbb{I}-\Pi_{M^{n}}\right)\left(v \cdot \nabla_{x} M^{n}\right)$ in the right-hand-side of 4.1) is discretized by a central difference scheme

$$
\left.\left(\mathbb{I}-\Pi_{M^{n}}\right)\left(v \partial_{x} M^{n}\right)\right|_{x_{i+\frac{1}{2}}} \approx\left(\mathbb{I}-\Pi_{i+\frac{1}{2}}^{n}\right)\left(v \delta^{0} M_{i+\frac{1}{2}}^{n}\right)
$$

where $\Pi_{i+\frac{1}{2}}^{n}$ is an approximation of $\Pi_{M\left(U\left(t_{n}, x_{i+\frac{1}{2}}\right)\right)}$. A suitable choice of $\Pi_{i+\frac{1}{2}}^{n}$ is given by $([2]$

$$
\Pi_{i+\frac{1}{2}}^{n}=\frac{\Pi_{i}^{n}+\Pi_{i+1}^{n}}{2}=\frac{\Pi\left(U_{i}^{n}\right)+\Pi\left(U_{i+1}^{n}\right)}{2}, \quad \text { or } \Pi_{i+\frac{1}{2}}=\Pi\left(\frac{U_{i}+U_{i+1}}{2}\right),
$$

and $M_{i+\frac{1}{2}}^{n} \approx \frac{M_{i}^{n}+M_{i+1}^{n}}{2}$.

I. For the Boltzmann equation, the discretized scheme of the microscopic equations 4.1 is given by

$$
\begin{aligned}
& \frac{g_{i+\frac{1}{2}}^{n+1}-g_{i+\frac{1}{2}}^{n}}{\Delta t}+\left(\mathbb{I}-\Pi_{i+\frac{1}{2}}^{n}\right)\left(v^{+} \frac{g_{i+\frac{1}{2}}^{n}-g_{i-\frac{1}{2}}^{n}}{\Delta x}+v^{-} \frac{g_{i+\frac{3}{2}}^{n}-g_{i+\frac{1}{2}}^{n}}{\Delta x}\right)-\mathcal{Q}_{B}\left(g_{i+\frac{1}{2}}^{n}, g_{i+\frac{1}{2}}^{n}\right) \\
= & \frac{1}{\varepsilon}\left[\frac{1}{2}\left(\mathcal{Q}_{B}\left(M_{i+\frac{1}{2}}^{n}+g_{i+\frac{1}{2}}^{n}, M_{i+\frac{1}{2}}^{n}+g_{i+\frac{1}{2}}^{n}\right)-\mathcal{Q}_{B}\left(M_{i+\frac{1}{2}}^{n}-g_{i+\frac{1}{2}}^{n}, M_{i+\frac{1}{2}}^{n}-g_{i+\frac{1}{2}}^{n}\right)\right)+\frac{1}{2}\left(\beta_{1}^{n}+\beta_{2}^{n}\right) g_{i+\frac{1}{2}}^{n}\right. \\
& \left.-\frac{1}{2}\left(\beta_{1}^{n+1}+\beta_{2}^{n+1}\right) g_{i+\frac{1}{2}}^{n+1}-\left(\mathbb{I}-\Pi_{i+\frac{1}{2}}^{n}\right)\left(v \frac{M_{i+1}^{n}-M_{i}^{n}}{\Delta x}\right)\right],
\end{aligned}
$$

thus

$$
\begin{aligned}
g_{i+\frac{1}{2}}^{n+1}=\frac{1}{1+\frac{\Delta t}{2 \varepsilon}\left(\beta_{1}^{n+1}+\beta_{2}^{n+1}\right)} & {\left[g_{i+\frac{1}{2}}^{n}-\Delta t\left(\mathbb{I}-\Pi_{i+\frac{1}{2}}^{n}\right)\left(v^{+} \frac{g_{i+\frac{1}{2}}^{n}-g_{i-\frac{1}{2}}^{n}}{\Delta x}+v^{-} \frac{g_{i+\frac{3}{2}}^{n}-g_{i+\frac{1}{2}}^{n}}{\Delta x}\right)\right.} \\
+\Delta t \mathcal{Q}_{B}\left(g_{i+\frac{1}{2}}^{n}, g_{i+\frac{1}{2}}^{n}\right)+ & \frac{\Delta t}{\varepsilon}\left(\frac{1}{2}\left(\mathcal{Q}_{B}\left(M_{i+\frac{1}{2}}^{n}+g_{i+\frac{1}{2}}^{n}, M_{i+\frac{1}{2}}^{n}+g_{i+\frac{1}{2}}^{n}\right)-\mathcal{Q}_{B}\left(M_{i+\frac{1}{2}}^{n}-g_{i+\frac{1}{2}}^{n}, M_{i+\frac{1}{2}}^{n}-g_{i+\frac{1}{2}}^{n}\right)\right)\right. \\
& \left.\left.+\frac{1}{2}\left(\beta_{1}^{n}+\beta_{2}^{n}\right) g_{i+\frac{1}{2}}^{n}-\left(\mathbb{I}-\Pi_{i+\frac{1}{2}}^{n}\right)\left(v \frac{M_{i+1}^{n}-M_{i}^{n}}{\Delta x}\right)\right)\right] .
\end{aligned}
$$


Remark 4.4. To improve to second-order spatial discretization of $v \partial_{x} g$ in the above equation, one uses a second order upwind (MUSCL) discretization in (4.16), and then (4.20) is replaced by

$$
\begin{aligned}
g_{i+\frac{1}{2}}^{n+1}=\frac{1}{1+\frac{\Delta t}{2 \varepsilon}\left(\beta_{1}^{n+1}+\beta_{2}^{n+1}\right)}\left[g_{i+\frac{1}{2}}^{n}-\Delta t\left(\mathbb{I}-\Pi_{i+\frac{1}{2}}^{n}\right)\left(\frac{G_{i+1}^{n}-G_{i}^{n}}{\Delta x}\right)\right. \\
+\Delta t \mathcal{Q}_{B}\left(g_{i+\frac{1}{2}}^{n}, g_{i+\frac{1}{2}}^{n}\right)+\frac{\Delta t}{\varepsilon}\left(\frac{1}{2}\left(\mathcal{Q}_{B}\left(M_{i+\frac{1}{2}}^{n}+g_{i+\frac{1}{2}}^{n}, M_{i+\frac{1}{2}}^{n}+g_{i+\frac{1}{2}}^{n}\right)-\mathcal{Q}_{B}\left(M_{i+\frac{1}{2}}^{n}-g_{i+\frac{1}{2}}^{n}, M_{i+\frac{1}{2}}^{n}-g_{i+\frac{1}{2}}^{n}\right)\right)\right. \\
\left.\left.+\frac{1}{2}\left(\beta_{1}^{n}+\beta_{2}^{n}\right) g_{i+\frac{1}{2}}^{n}-\left(\mathbb{I}-\Pi_{i+\frac{1}{2}}^{n}\right)\left(v \frac{M_{i+1}^{n}-M_{i}^{n}}{\Delta x}\right)\right)\right]
\end{aligned}
$$

where

$G_{i}^{n}=v^{+} g_{i}^{+, n}+v^{-} g_{i}^{-, n}=v^{+}\left(g_{i-\frac{1}{2}}^{n}+\frac{\Delta x}{2} \delta g_{i-\frac{1}{2}}^{n}\right)+v^{-}\left(g_{i+\frac{1}{2}}^{n}-\frac{\Delta x}{2} \delta g_{i+\frac{1}{2}}^{n}\right), \quad i=0, \cdots, N$,

and $\delta g$ represents a slope with a slope limiter, given by [20]

$$
\delta g_{j-\frac{1}{2}}^{n}=\frac{1}{\Delta x} \operatorname{minmod}\left\{g_{j+\frac{1}{2}}^{n}-g_{j-\frac{1}{2}}^{n}, g_{j-\frac{1}{2}}^{n}-g_{j-\frac{3}{2}}^{n}\right\}, \quad j=0, \cdots, N+1 .
$$

II. For the nFPL equation, we first introduce the symmetrized operator in 15 ]

$$
\widetilde{P} h=\frac{1}{\sqrt{M}} \nabla_{v} \cdot\left(M \nabla_{v}\left(\frac{h}{\sqrt{M}}\right)\right) .
$$

Thus the penalty operator given in 4.7 can be rewritten as

$$
P_{F P}^{M} f=\sqrt{M} \widetilde{P} \frac{f}{\sqrt{M}} .
$$

Use 4.8, 4.19 correspondingly becomes

$$
\begin{aligned}
g_{i+\frac{1}{2}}^{n+1}=\left(\mathbb{I}-\frac{\Delta t}{2 \varepsilon}\left(\beta_{1}^{n}+\beta_{2}^{n}\right) P^{n+1}\right)^{-1}\left[g_{i+\frac{1}{2}}^{n}-\Delta t\left(\mathbb{I}-\Pi_{i+\frac{1}{2}}^{n}\right)\left(v^{+} \frac{g_{i+\frac{1}{2}}^{n}-g_{i-\frac{1}{2}}^{n}}{\Delta x}+v^{-} \frac{g_{i+\frac{3}{2}}^{n}-g_{i+\frac{1}{2}}^{n}}{\Delta x}\right)\right. \\
+\Delta t \mathcal{Q}_{L}\left(g_{i+\frac{1}{2}}^{n}, g_{i+\frac{1}{2}}^{n}\right)+\frac{\Delta t}{\varepsilon}\left(\frac{1}{2}\left(\mathcal{Q}_{L}\left(M_{i+\frac{1}{2}}^{n}+g_{i+\frac{1}{2}}^{n}, M_{i+\frac{1}{2}}^{n}+g_{i+\frac{1}{2}}^{n}\right)-\mathcal{Q}_{L}\left(M_{i+\frac{1}{2}}^{n}-g_{i+\frac{1}{2}}^{n}, M_{i+\frac{1}{2}}^{n}-g_{i+\frac{1}{2}}^{n}\right)\right)\right. \\
\left.\left.-\frac{1}{2}\left(\beta_{1}^{n}+\beta_{2}^{n}\right) P^{n} g_{i+\frac{1}{2}}^{n}-\left(\mathbb{I}-\Pi_{i+\frac{1}{2}}^{n}\right)\left(v \frac{M_{i+1}^{n}-M_{i}^{n}}{\Delta x}\right)\right)\right] .
\end{aligned}
$$

Rewrite the above equation 4.23 as

$$
\begin{aligned}
\left(\frac{g_{i+\frac{1}{2}}}{\sqrt{M}}\right)^{n+1}= & \left(\mathbb{I}-\frac{\Delta t}{2 \varepsilon}\left(\beta_{1}^{n}+\beta_{2}^{n}\right) \widetilde{P}^{n+1}\right)^{-1}\left\{\frac { 1 } { \sqrt { M ^ { n + 1 } } } \left[g_{i+\frac{1}{2}}^{n}-\Delta t\left(\mathbb{I}-\Pi_{i+\frac{1}{2}}^{n}\right)\right.\right. \\
& \cdot\left(v^{+} \frac{g_{i+\frac{1}{2}}^{n}-g_{i-\frac{1}{2}}^{n}}{\Delta x}+v^{-} \frac{g_{i+\frac{3}{2}}^{n}-g_{i+\frac{1}{2}}^{n}}{\Delta x}\right)+\Delta t \mathcal{Q}_{L}\left(g_{i+\frac{1}{2}}^{n}, g_{i+\frac{1}{2}}^{n}\right) \\
+ & \frac{\Delta t}{\varepsilon}\left(\frac{1}{2}\left(\mathcal{Q}_{L}\left(M_{i+\frac{1}{2}}^{n}+g_{i+\frac{1}{2}}^{n}, M_{i+\frac{1}{2}}^{n}+g_{i+\frac{1}{2}}^{n}\right)-\mathcal{Q}_{L}\left(M_{i+\frac{1}{2}}^{n}-g_{i+\frac{1}{2}}^{n}, M_{i+\frac{1}{2}}^{n}-g_{i+\frac{1}{2}}^{n}\right)\right)\right. \\
& \left.\left.\left.\quad-\frac{1}{2}\left(\beta_{1}^{n}+\beta_{2}^{n}\right) \sqrt{M^{n}} \widetilde{P} \frac{g_{i+\frac{1}{2}}^{n}}{\sqrt{M^{n}}}-\left(\mathbb{I}-\Pi_{i+\frac{1}{2}}^{n}\right)\left(v \frac{M_{i+1}^{n}-M_{i}^{n}}{\Delta x}\right)\right)\right]\right\} .
\end{aligned}
$$


One can apply the Conjugate Gradient (CG) method to get $\left(\frac{g_{i+\frac{1}{2}}}{\sqrt{M}}\right)^{n+1}$, which is used in [15].

A second order discretization of $\left(\mathbb{I}-\Pi_{M^{n}}\right)\left(v \partial_{x} g^{n}\right)$ can also be used as in 4.22).

Velocity discretization of $\widetilde{P}$ As was done in [15], the discretization of $\tilde{P}$ in one dimension is given by

$$
\begin{aligned}
(\tilde{P} h)_{j} & =\frac{1}{(\Delta v)^{2}} \frac{1}{\sqrt{M_{j}}}\left\{\sqrt{M_{j} M_{j+1}}\left(\left(\frac{h}{\sqrt{M}}\right)_{j+1}-\left(\frac{h}{\sqrt{M}}\right)_{j}\right)-\sqrt{M_{j} M_{j-1}}\left(\left(\frac{h}{\sqrt{M}}\right)_{j}-\left(\frac{h}{\sqrt{M}}\right)_{j-1}\right)\right\} \\
& =\frac{1}{(\Delta v)^{2}}\left(h_{j+1}-\frac{\sqrt{M_{j+1}}+\sqrt{M_{j-1}}}{\sqrt{M_{j}}} h_{j}+h_{j-1}\right) .
\end{aligned}
$$

It is obvious that $\tilde{P}$ is symmetric. We discretize dimension-by-dimension in velocity space.

\subsection{The Asymptotic-Preserving property of the scheme}

In this section, we investigate the formal fluid dynamics behavior (for $\varepsilon \ll 1$ ) of the discretized numerical scheme given by 4.20 and 4.12 for the Boltzmann equation, in order to show that the scheme is Asymptotic-Preserving (AP) [12, 13] in the fluid dynamic regime. For notation simplicity, rewrite the term

$$
\mathcal{L}_{M_{i+\frac{1}{2}}^{n}}\left(g_{i+\frac{1}{2}}^{n}\right)=\frac{1}{2}\left(\mathcal{Q}\left(M_{i+\frac{1}{2}}^{n}+g_{i+\frac{1}{2}}^{n}, M_{i+\frac{1}{2}}^{n}+g_{i+\frac{1}{2}}^{n}\right)-\mathcal{Q}\left(M_{i+\frac{1}{2}}^{n}-g_{i+\frac{1}{2}}^{n}, M_{i+\frac{1}{2}}^{n}-g_{i+\frac{1}{2}}^{n}\right)\right) .
$$

From the right hand side of 4.19, one can see

$$
\begin{aligned}
\mathcal{L}_{M_{i+\frac{1}{2}}^{n}}\left(g_{i+\frac{1}{2}}^{n}\right) & +\frac{1}{2}\left(\beta_{1}^{n}+\beta_{2}^{n}\right) g_{i+\frac{1}{2}}^{n}-\frac{1}{2}\left(\beta_{1}^{n+1}+\beta_{2}^{n+1}\right) g_{i+\frac{1}{2}}^{n+1} \\
& -\left(\mathbb{I}-\Pi_{i+\frac{1}{2}}^{n}\right)\left(v \frac{M_{i+1}^{n}-M_{i}^{n}}{\Delta x}\right)=O(\varepsilon) .
\end{aligned}
$$

We make the following assumptions similar to that in [6]: there exists a constant $C>0$ such that

$$
\left|g^{n}\right|+\left|\frac{g^{n+1}-g^{n}}{\Delta t}\right| \leq C
$$

and

$$
\left|U^{n}\right|+\left|\frac{U^{n+1}-U^{n}}{\Delta t}\right| \leq C
$$

These are typical assumptions for AP schemes, since the fluid dynamic limit of the Boltzmann or FPL equation is not rigorously justified even in the continuous case when solutions admit singularities such as shocks.

Denote $\beta=\frac{1}{2}\left(\beta_{1}+\beta_{2}\right)$, we have in 4.26

$$
\begin{aligned}
\operatorname{term} I & :=\frac{1}{2}\left(\beta_{1}^{n}+\beta_{2}^{n}\right) g_{i+\frac{1}{2}}^{n}-\frac{1}{2}\left(\beta_{1}^{n+1}+\beta_{2}^{n+1}\right) g_{i+\frac{1}{2}}^{n+1}=\beta^{n} g_{i+\frac{1}{2}}^{n}-\beta^{n+1} g_{i+\frac{1}{2}}^{n+1} \\
& =\beta^{n+1}\left(g_{i+\frac{1}{2}}^{n}-g_{i+\frac{1}{2}}^{n+1}\right)+\left(\beta^{n}-\beta^{n+1}\right) g_{i+\frac{1}{2}}^{n} .
\end{aligned}
$$

Under the assumption 4.27) and 4.28, and since $\beta^{n}$ only depends on $U^{n}$, one gets

$$
|\operatorname{term} I|=O(\Delta t)
$$

From 4.26, $g_{i+\frac{1}{2}}^{n}$ is approximated by

$$
g_{i+\frac{1}{2}}^{n}=\mathcal{L}_{M_{i+\frac{1}{2}}^{n}}^{-1}\left\{\left(\mathbb{I}-\Pi_{i+\frac{1}{2}}^{n}\right)\left(v \frac{M_{i+1}^{n}-M_{i}^{n}}{\Delta x}\right)\right\}+O(\varepsilon)+O(\Delta t) .
$$


$g_{i+\frac{1}{2}}^{n+1}$ can be approximated by $g_{i+\frac{1}{2}}^{n}+O(\Delta t)$, thus

$$
g_{i+\frac{1}{2}}^{n+1}=\mathcal{L}_{M_{i+\frac{1}{2}}^{n}}^{-1}\left\{\left(\mathbb{I}-\Pi_{i+\frac{1}{2}}^{n}\right)\left(v \frac{M_{i+1}^{n}-M_{i}^{n}}{\Delta x}\right)\right\}+O(\varepsilon)+O(\Delta t) .
$$

Plug 4.30 into 4.12,

$$
\begin{aligned}
& \frac{U_{i}^{n+1}-U_{i}^{n}}{\Delta t}+\frac{F_{i+\frac{1}{2}}\left(U^{n}\right)-F_{i-\frac{1}{2}}\left(U^{n}\right)}{\Delta x}=\frac{\varepsilon}{\Delta x}\left\langlev m \left\{\mathcal{L}_{M_{i+\frac{1}{2}}^{n}}^{-1}\left[\left(\mathbb{I}-\Pi_{i+\frac{1}{2}}^{n}\right)\left(v \frac{M_{i+1}^{n}-M_{i}^{n}}{\Delta x}\right)\right]\right.\right. \\
& \left.\left.-\mathcal{L}_{M_{i-\frac{1}{2}}^{n}}^{-1}\left[\left(\mathbb{I}-\Pi_{i-\frac{1}{2}}^{n}\right)\left(v \frac{M_{i}^{n}-M_{i-1}^{n}}{\Delta x}\right)\right]\right\}\right\rangle+O\left(\varepsilon \Delta t+\varepsilon^{2}\right) .
\end{aligned}
$$

Following the same calculation as [2, 6], one obtains

$$
\left(\mathbb{I}-\Pi_{M}\right)\left(v \cdot \nabla_{x} M\right)=\left(B:\left(\nabla_{x} u+\left(\nabla_{x} u\right)^{T}-\frac{2}{d}\left(\nabla_{x} \cdot u\right) \mathbb{I}\right)+A \cdot \frac{\nabla_{x} T}{\sqrt{T}}\right) M+O(\varepsilon),
$$

where

$$
A=\left(\frac{|v-u|^{2}}{2 T}-\frac{d+2}{2}\right) \frac{v-u}{\sqrt{T}}, \quad B=\frac{1}{2}\left(\frac{(v-u) \otimes(v-u)}{2 T}-\frac{|v-u|^{2}}{d T} \mathbb{I}\right) .
$$

Therefore,

$\mathcal{L}_{M^{n}}^{-1}\left(\left(\mathbb{I}-\Pi_{M^{n}}\right)\left(v \cdot \nabla_{x} M^{n}\right)\right)=\mathcal{L}_{M^{n}}^{-1}(B M):\left(\nabla_{x} u+\left(\nabla_{x} u\right)^{T}-\frac{2}{d}\left(\nabla_{x} \cdot u\right) \mathbb{I}\right)+\mathcal{L}_{M^{n}}^{-1}(A M) \cdot \frac{\nabla_{x} T}{\sqrt{T}}$.

Thus 4.31 is a consistent time discretization scheme to the compressible Navier-Stokes system, with the order of $\varepsilon$ term given by

$$
\varepsilon \nabla_{x} \cdot\left(\begin{array}{c}
0 \\
\mu \sigma(u) \\
\mu \sigma(u) u+\kappa \nabla_{x} T
\end{array}\right)
$$

with

$$
\sigma(u)=\nabla_{x} u+\left(\nabla_{x} u\right)^{T}-\frac{2}{d} \nabla_{x} \cdot u \mathbb{I},
$$

where the viscosity $\mu=\mu(T)$ and the thermal conductivity $\kappa=\kappa(T)$ only depend on the temperature and whose general expressions can be found in [1.

We summarize the conclusions in the following theorem. Compared to Proposition 4.3 in [2], the result here is valid for the full Boltzmann instead of the BGK equation.

Theorem 4.5. Consider the time and space discretizations of the Boltzmann equation, given by equation (4.20) and 4.12), then

(i) In the limit $\varepsilon \rightarrow 0$, the moments $U^{n}$ satisfy the following discretization of the Euler equations

$$
\frac{U_{i}^{n+1}-U_{i}^{n}}{\Delta t}+\frac{F_{i+\frac{1}{2}}\left(U^{n}\right)-F_{i-\frac{1}{2}}\left(U^{n}\right)}{\Delta x}=0 .
$$

(ii) The scheme 4.20) and 4.12) is asymptotically equivalent, with an error of $O\left(\varepsilon^{2}\right)$, to the following scheme,

$$
\begin{array}{r}
\frac{U_{i}^{n+1}-U_{i}^{n}}{\Delta t}+\frac{F_{i+\frac{1}{2}}\left(U^{n}\right)-F_{i-\frac{1}{2}}\left(U^{n}\right)}{\Delta x}=\frac{\varepsilon}{\Delta x}\left\langlev m \left\{\mathcal{L}_{M_{i+\frac{1}{2}}^{n}}^{-1}\left[\left(\mathbb{I}-\Pi_{i+\frac{1}{2}}^{n}\right)\left(v \frac{M_{i+1}^{n}-M_{i}^{n}}{\Delta x}\right)\right]\right.\right. \\
\quad-\mathcal{L}_{M_{i-\frac{1}{2}}^{n}}^{-1}\left[\left(\mathbb{I}-\Pi_{i-\frac{1}{2}}^{n}\right)\left(v \frac{\left.\left.\left.\left.M_{i}^{n}-M_{i-1}^{n}\right)\right]\right\}\right\rangle,}{\Delta x}\right)\right]
\end{array}
$$

which is a consistent approximation of the compressible Navier-Stokes equation, provided that the viscous terms are resolved numerically.

From (ii), it shows that one needs the mesh size and time step to be $O(\varepsilon)$ in order to capture the Navier-Stokes approximation. This is necessary for any scheme since the viscosity and heat conductivity are of $O(\varepsilon)$. 


\section{$5 \quad$ Numerical Implementation}

We mention some details in the numerical implementation. Assume we have all the values of $U$ and $g$ at time $t^{n}$, namely $g_{-\frac{1}{2}}^{n}, \cdots, g_{N+\frac{1}{2}}^{n}$, and $U_{-1}^{n}, U_{0}^{n}, \cdots, U_{N+1}^{n}, U_{N+2}^{n}$.

(i) Step 1. $g$ is calculated at staggered grids $x_{\frac{1}{2}}, \cdots, x_{N-\frac{1}{2}}$.

We use equation 4.20 for the Boltzmann or 4.23 for the Landau equation (with a rewritten form of (4.24) ). The projection operator is given in 4.18). Here the second choice is used. Denote

$$
M^{*}=\frac{M\left(U_{i}\right)+M\left(U_{i+1}\right)}{2}
$$

by definition of $\Pi$, one has

$$
\Pi_{M^{*}}(\psi)=\frac{1}{\rho}\left[\langle\psi\rangle+\frac{(v-u) \cdot\langle(v-u) \psi\rangle}{T}+\left(\frac{|v-u|^{2}}{2 T}-\frac{d}{2}\right) \frac{2}{d}\left\langle\left(\frac{|v-u|^{2}}{2 T}-\frac{d}{2}\right) \psi\right\rangle\right] M^{*},
$$

where $\rho, u, T$ are associated with $M^{*}$ as in 2.8. If one assumes the periodic in $x$ boundary condition, then

$$
g_{-\frac{1}{2}}=g_{N-\frac{1}{2}}, \quad g_{N+\frac{1}{2}}=g_{\frac{1}{2}} .
$$

Free-flow boundary condition is used in the shock-tube tests, that is,

$$
g_{-\frac{1}{2}}=g_{\frac{1}{2}}, \quad g_{N+\frac{1}{2}}=g_{N-\frac{1}{2}},
$$

and similarly for $U$.

(ii) Step 2. $U$ is calculated at $i=1, \cdots, N$, by using 4.12 , where values of $g_{\frac{1}{2}}^{n+1}, \cdots, g_{N+\frac{1}{2}}^{n+1}$ are used. The numerical flux $F$ is calculated by first or second order splitting with slope limiters. We apply a second-order TVD method. Following [2], we use a simple reconstruction of the upwind flux $F_{i+\frac{1}{2}}\left(U^{n}\right)(i=0, \cdots, N)$ from the flux splitting that is naturally derived from its kinetic formulation:

$$
F(U)=\left\langle v^{+} m M(U)\right\rangle+\left\langle v^{-} m M(U)\right\rangle:=F^{+}(U)+F^{-}(U) .
$$

A second order approximation of the positive and negative flux is obtained by a linear piecewise polynomial $\hat{F}_{i}$ for $i=0, \cdots, N+1$. Then we reconstruct the numerical flux $F_{i+\frac{1}{2}}(U)(i=0, \cdots, N)$ in a split form,

$$
F_{i+\frac{1}{2}}^{n}=F^{+}\left(U_{i}^{n}\right)+s_{i}^{+, n} \frac{\Delta x}{2}+F^{-}\left(U_{i+1}^{n}\right)-s_{i+1}^{-, n} \frac{\Delta x}{2},
$$

where a slope limiter $s_{i}^{ \pm, n}$ is introduced to suppress possible spurious oscillations near discontinuities. We use a second order TVD minmod slope limiter [20],

$$
s_{i}^{ \pm, n}=\frac{1}{\Delta x} \operatorname{minmod}\left\{F^{ \pm}\left(U_{i+1}^{n}\right)-F^{ \pm}\left(U_{i}^{n}\right), F^{ \pm}\left(U_{i}^{n}\right)-F^{ \pm}\left(U_{i-1}^{n}\right)\right\} .
$$

Note that we need $F\left(U_{-1}\right), F\left(U_{0}\right), F\left(U_{N+1}\right), F\left(U_{N+2}\right)$ when computing $s_{0}^{+}, s_{N+1}^{-}$, thus two ghost cells are needed. For periodic BC, we let

$$
U_{0}=U_{N}, \quad U_{-1}=U_{N-1}, \quad U_{N+1}=U_{1}, \quad U_{N+2}=U_{2} .
$$

Implementation details of solving 4.2 are shown in the Appendix. 


\section{Numerical Examples}

Test I: The micro-macro scheme for the Boltzmann equation

Consider the spatial variable $x \in[0,1]$. Periodic boundary condition is used except for the shock tube tests. The velocity variable $v \in\left[-L_{v}, L_{v}\right]^{2}$ with $L_{v}=8.4 . \quad N_{x}=100$, $\Delta t=\Delta x / 20$. Note that the velocity domain should be chosen large enough so that the numerical solution $f$ is essentially zero at its boundary. The fast spectral method in [31] is applied to evaluate the collision operator $\mathcal{Q}$ and 32 points are used in each velocity dimension.

In order to compare different schemes, we denote by ' $\mathrm{FJ}$ ' the Filbet-Jin AP method with penalty proposed in [6] for the Boltzmann equation; by 'JY' the Jin-Yan AP method with penalty in [15] for the Landau equation. 'MM' stands for the micro-macro scheme for the full Boltzmann and Landau equations we propose in the current paper. 'DS' represents a direct, explicit 4th order Runge-Kutta time discretization solver for the Boltzmann or Landau equations.

\section{Test I (a)}

The initial data is given by

$$
\rho^{0}(x)=\frac{2+\sin (2 \pi x)}{3}, \quad u^{0}=(0.2,0), \quad T^{0}(x)=\frac{3+\cos (2 \pi x)}{4} .
$$

The following non-equilibrium double-peak initial distribution is considered,

$$
f^{0}(x, v)=\frac{\rho^{0}}{4 \pi T^{0}}\left(e^{-\frac{\left|v-u^{0}\right|^{2}}{2 T^{0}}}+e^{-\frac{\left|v+u^{0}\right|^{2}}{2 T^{0}}}\right) .
$$

Test I (b)

In this example, we consider a mixed regime with the Knudsen number $\varepsilon$ varying in space, where $x \in[0,1], v \in[-6,6]^{2}$,

$$
\varepsilon(x)=\left\{\begin{array}{l}
10^{-2}+\frac{1}{2}(\tanh (25-20 x)+\tanh (-5+20 x)), \quad x \leq 0.65 \\
10^{-2}, \quad x>0.65
\end{array}\right.
$$

The initial data is given by $6.1-6.2$.

Test I (c). We study a Sod shock tube test problem for the Boltzmann equation. The equilibrium initial distribution is given by

$$
f^{0}(x, v)=\frac{\rho^{0}}{2 \pi T^{0}} e^{-\frac{\left|v-u^{0}\right|^{2}}{2 T^{0}}}
$$

where the initial data for $\rho^{0}, u^{0}$ and $T^{0}$ are given by

$$
\left\{\begin{array}{l}
\rho_{l}=1, \quad u_{l}=(0,0), \quad T_{l}=1, \quad x \leq 0.5, \\
\rho_{r}=0.125, \quad u_{r}=(0,0), \quad T_{r}=0.25, \quad x>0.5 .
\end{array}\right.
$$

There are different choices of the free parameter $\beta$ in the penalty operators. We list below:

Choice 1. In the BGK penalty operator, $P=\beta(M-f)$, where $\beta$ is a positive constant chosen for stability. One can split the collision operator $\mathcal{Q}$ into the gaining part and the losing part $\mathcal{Q}(f, f)=\mathcal{Q}^{+}-f \mathcal{Q}^{-}$. In order to obtain positivity, it is sufficient to require $\beta>\mathcal{Q}^{-}([14)$. In our case,

$$
\beta_{1}^{n}>\mathcal{Q}_{1}^{-}, \quad \beta_{2}^{n}>\mathcal{Q}_{2}^{-},
$$


where $\mathcal{Q}_{1}, \mathcal{Q}_{2}$ represent the collision operator $\mathcal{Q}\left(M^{n}+g^{n}, M^{n}+g^{n}\right)$ and $\mathcal{Q}\left(M^{n}-g^{n}, M^{n}-g^{n}\right)$ respectively. Here $\beta_{1}^{n}, \beta_{2}^{n}$ are space and time dependent.

Choice 2. Another choice is given in [6], recall (4.6), we let

$$
\begin{aligned}
& \beta_{1}^{n}=\sup _{v}\left|\frac{\mathcal{Q}\left(M^{n}+g^{n}, M^{n}+g^{n}\right)}{g^{n}}\right|, \\
& \beta_{2}^{n}=\sup _{v}\left|\frac{\mathcal{Q}\left(M^{n}-g^{n}, M^{n}-g^{n}\right)}{g^{n}}\right| .
\end{aligned}
$$

Now we present and compare numerical results using difference schemes. Property of conservation of moments will also be verified. In the figure titles, $P_{0}, P_{1}, P_{2}$ represent the mass, momentum (in $v_{1}$ direction) and the total energy respectively. For Test I (a), Figure 1 (for $\varepsilon=1$ ) and Figure 2 (for $\varepsilon=10^{-4}$ ) show the time evolution of mass, momentum and energy obtained from $f$ (using $f=\mathcal{M}+\varepsilon g$ ), denoted by 'Mf' (see Remark 4.3), and from solving the macroscopic equations, denoted by 'ME' (moment equations) below. Figure 1 uses 'DS' and Figure 2 uses 'MM' for small $\varepsilon$. One can observe that the moments calculated from 'ME' are perfectly conserved with values unchanged as time propagates, while the conservation is not guaranteed if the moments are obtained from $f$ itself, however Figure 9 later shows that the error in total energy conservation is bounded for long time. This phenomenon verifies the proof that moments solved from ' $\mathrm{ME}$ ' are conserved as shown in 4.15. Moments computed from $f$, although not exactly conserved, however owes an spectral accuracy due to the numerical error of the spectral method used for the collision operators. One observes that if not using the moments systems to obtain the conserved quantities, third moments usually own a larger error than lower (first and second) moments by using the same discretization, a phenomenon that is also observed in several other tests in the following sections. The reason might be due to that the error in $f$ is enlarged more when multiplying by $|v|^{2}$ (instead of 1 or $v$ ) in the integration to get third moments.

In Section 7 we will use this idea to obtain the conservative solvers for more general kinetic equations and for general numerical schemes, not just the micro-macro decomposition based [2] or penalty based [6, 15] approaches.

The density $\rho$, bulk velocity $u_{1}$ and temperature $T$ defined as the following:

$$
\rho=\int_{\mathbb{R}^{2}} f d v, u_{i}=\frac{1}{\rho} \int_{\mathbb{R}^{2}} v_{i} f d v(i=1,2), T=\frac{1}{2 \rho} \int_{\mathbb{R}^{2}}(v-u)^{2} f d v .
$$

The numerical solutions are shown in Figure 3 for Test I (a). Here $u_{2}=0$ and we omit plotting it. MM uses the penalty parameters in Choice 1. One can observe that the two different approaches 'FJ' and 'MM' are consistent and produce the same results.

For Test $\mathrm{I}(\mathrm{b})$, the function $\varepsilon$ is plotted in Figure 4, whose values range from $10^{-2}$ to 1 , and is discontinuous at $x=0.65$. Figure 5 shows that by comparing with the 'DS' solutions as a reference, 'MM' is able to capture the macroscopic behavior efficiently with coarse mesh size and time steps when $\varepsilon$ is discontinuous, by using the penalty parameter $\beta$ in Choice 1 .

One can observe from Figure 6, for Test I (c), that the macroscopic quantities are well approximated although the mesh size and time steps are larger than $\varepsilon$, by using both the 'FJ' and 'MM' schemes, which give similar numerical results for the Sod problem. 

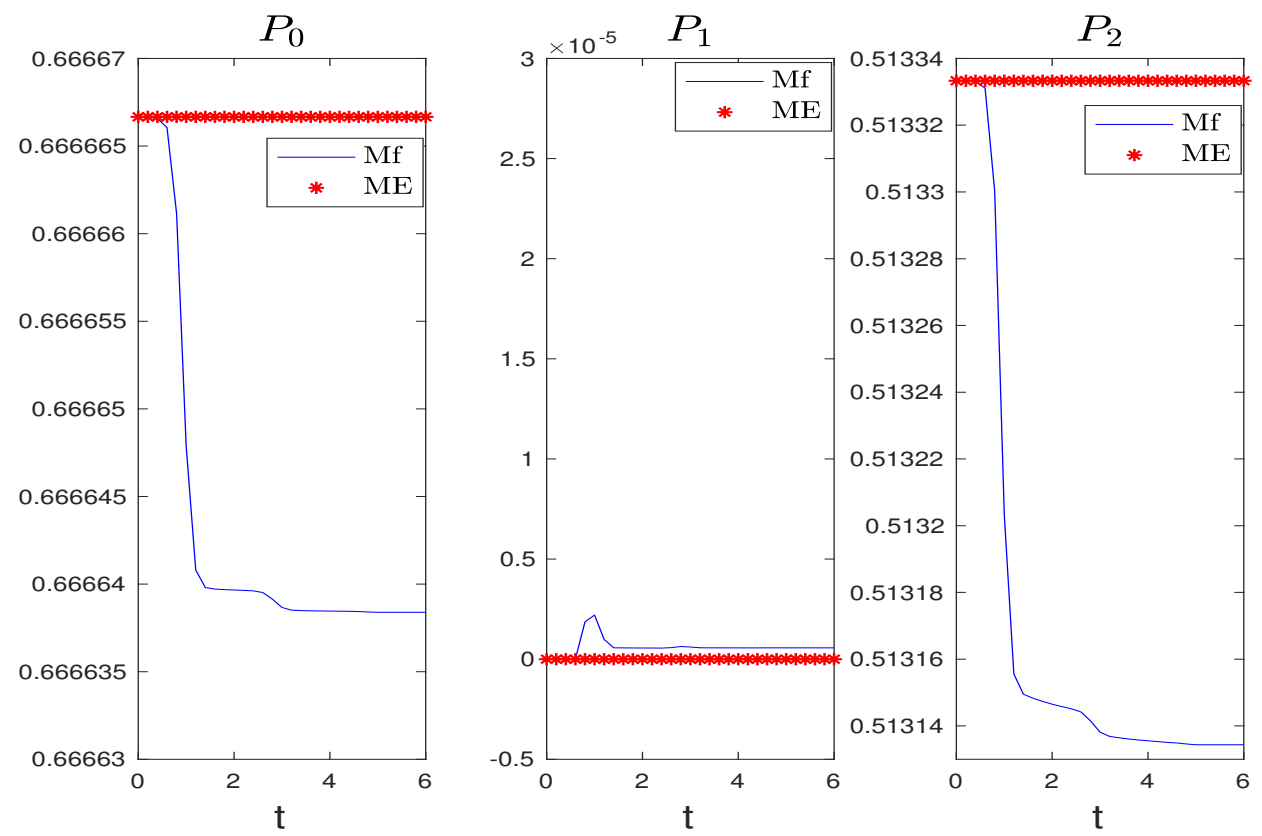

Figure 1: Test I (a). Time evolution of mass, momentum and energy by DS. 'Mf' versus 'ME'. $\varepsilon=1$.
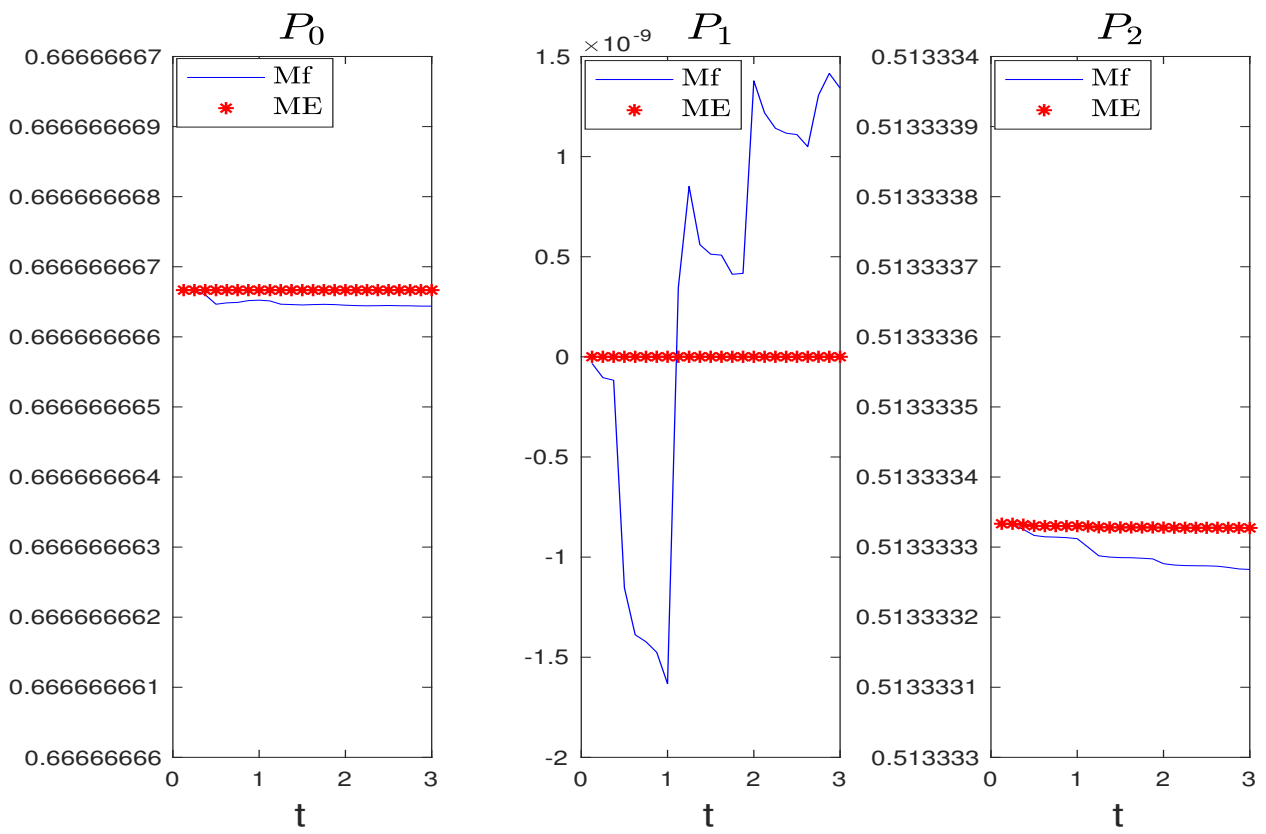

Figure 2: Test I (a). Time evolution of mass, momentum and energy by MM: 'Mf' versus 'ME'. $\varepsilon=10^{-4}$. 

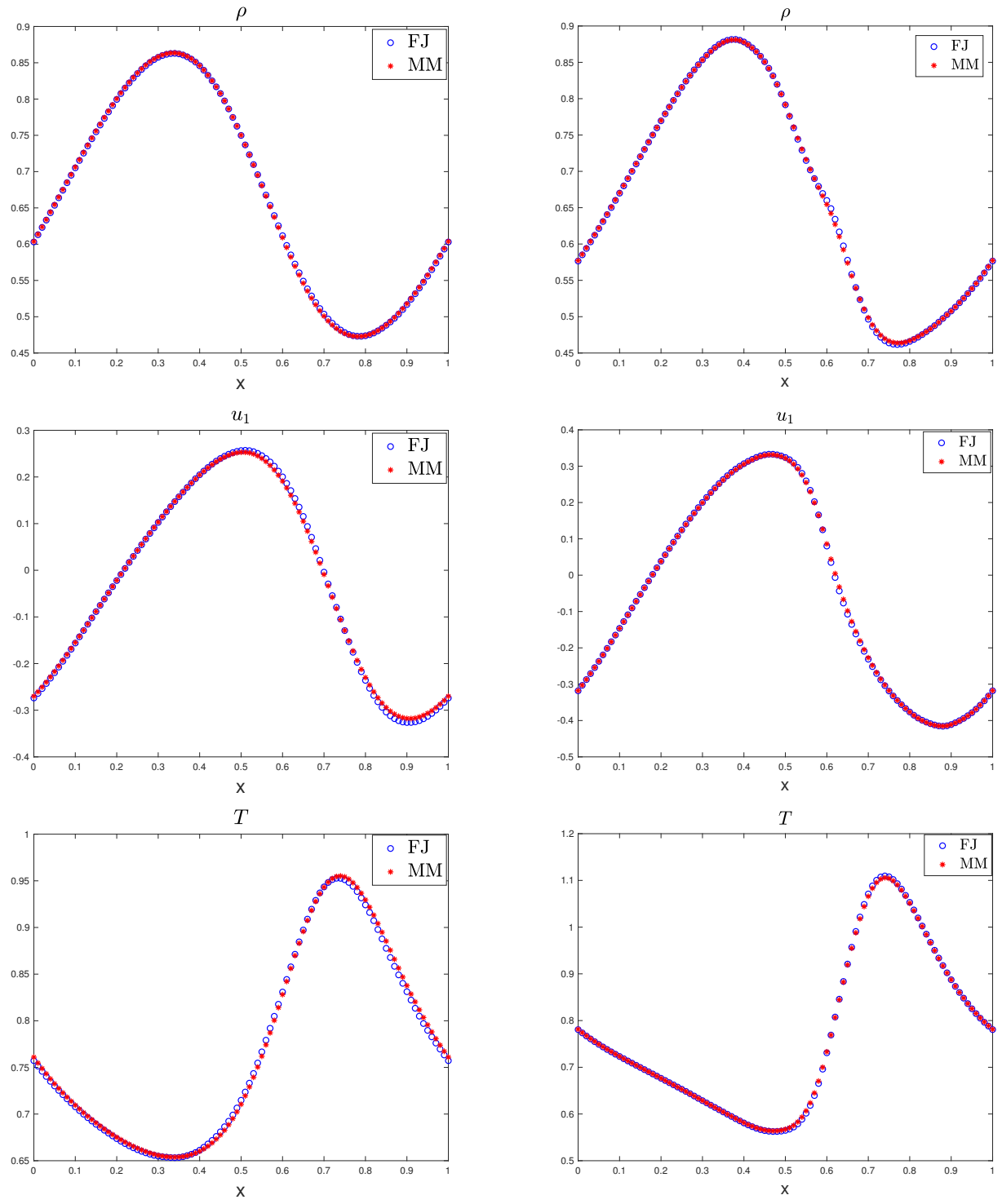

Figure 3: Test I (a). $t=0.2$. First column: $\varepsilon=1$. Second column: $\varepsilon=10^{-3}$. 


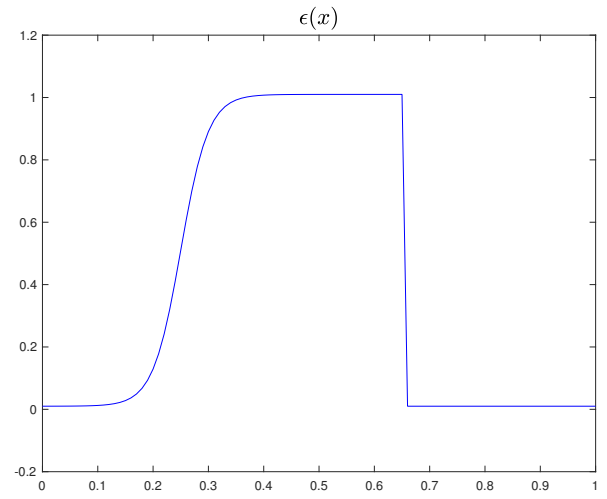

Figure 4: A spatially varying Knudsen number $\varepsilon(x)$ for Test I (b).
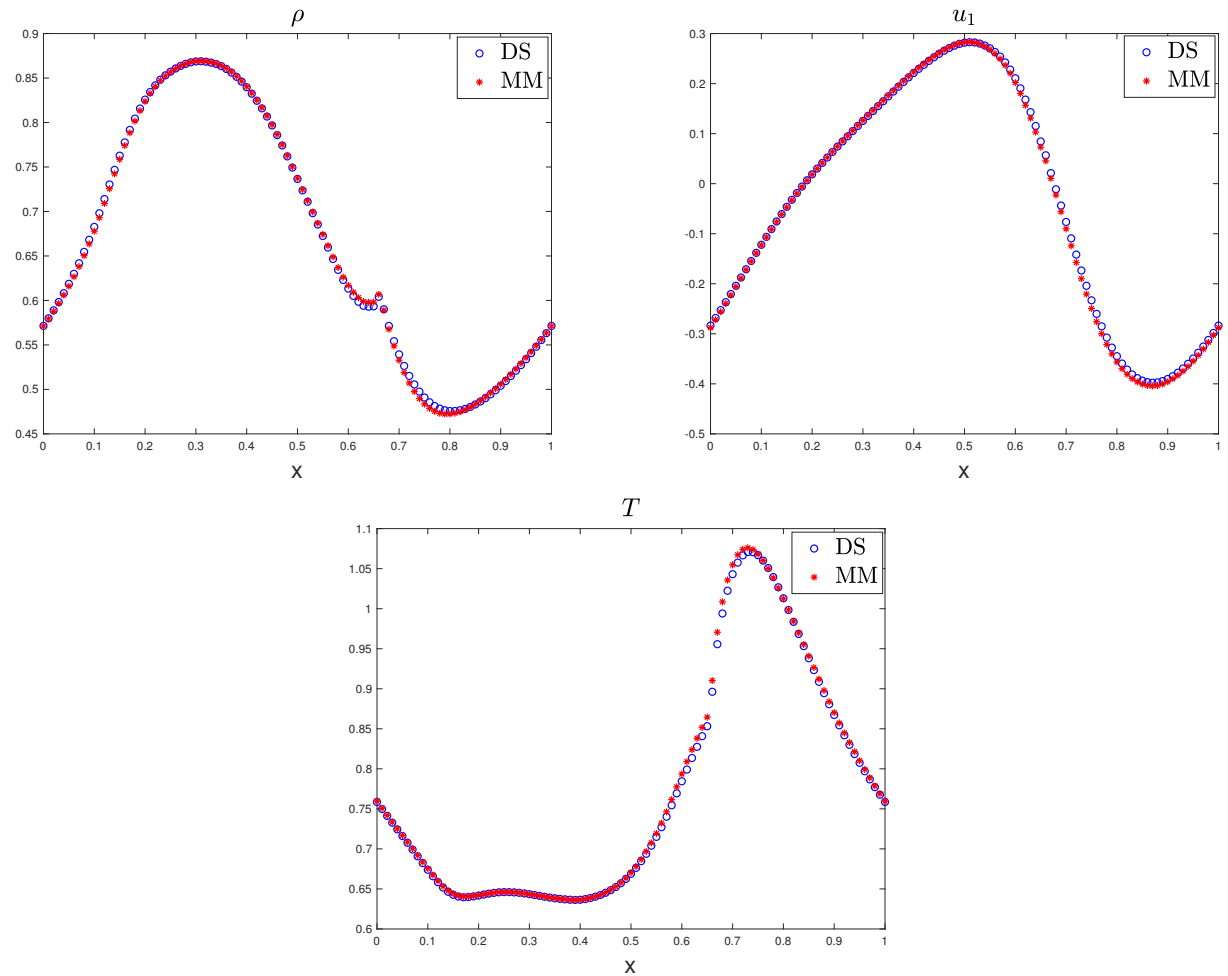

Figure 5: Test I (b). Numerical solutions at $t=0.2$ by 'DS' and 'MM'. 

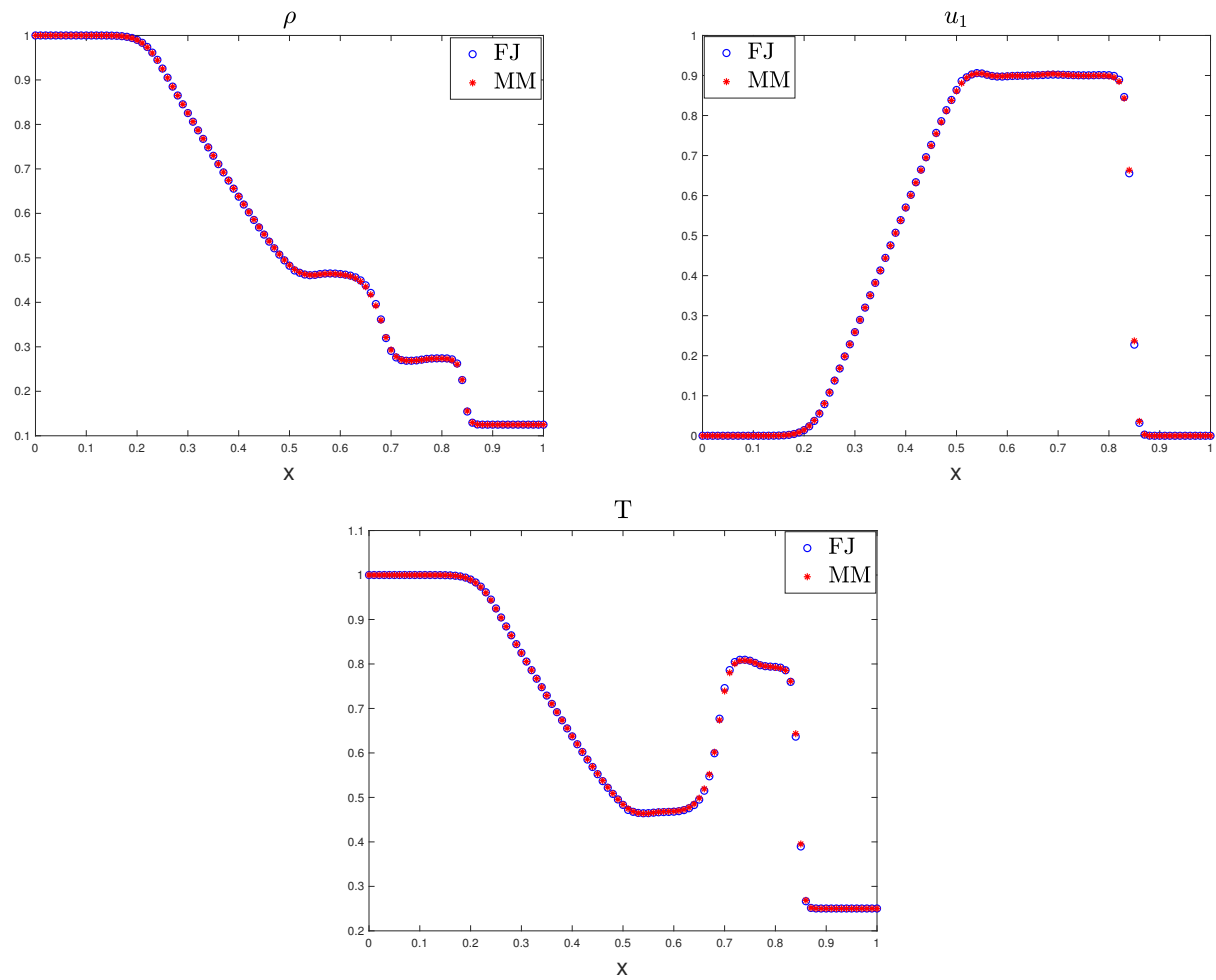

Figure 6: Test I (c). 'FJ' versus 'MM'. Numerical solutions at $t=0.2 . \varepsilon=10^{-4}$.

Test II: The micro-macro scheme for the nFPL equation

Test II (a). The initial data is given by

$$
\rho^{0}(x)=\frac{2+\sin (\pi x)}{3}, \quad u^{0}=(0.2,0), \quad T^{0}(x)=\frac{3+\cos (\pi x)}{4} .
$$

Consider the double-peak initial distribution $\left[6.2\right.$. Let $x \in[-1,1], v \in[-6,6]^{2}$ and $N_{x}=$ $100, N_{v}=32, \Delta t=\Delta x / 20 . \varepsilon=1$.

Test II (b).

We consider a Sod shock tube test for the nFPL equation with an equilibrium initial distribution:

$$
f^{0}(x, v)=\frac{\rho^{0}}{2 \pi T^{0}} e^{-\frac{\left|v-u^{0}\right|^{2}}{2 T^{0}}},
$$

where the initial data for $\rho^{0}, u^{0}$ and $T^{0}$ are given by

$$
\begin{array}{lr}
\left(\rho, u_{1}, T\right)=(1,0,1), \quad \text { if }-0.5 \leq x<0, \\
\left(\rho, u_{1}, T\right)=(0.125,0,0.25), & \text { if } 0 \leq x \leq 0.5 .
\end{array}
$$

Let $x \in[-0.5,0.5], v \in[-6,6]^{2}$ and $N_{x}=100, N_{v}=32, \Delta t=\Delta x / 20 . \varepsilon=10^{-3}$.

Figure 7 shows the numerical solutions of Test II (a) by 'MM' compared with 'DS', for both $\mathcal{O}(1)$ or moderately small $\varepsilon$, in good agreement. In Figure 8 for Test II (b), one can see that the macroscopic quantities are well approximated for the Sod shock tube test for the nFPL equation, although the mesh size and time steps are coarse, thus it verifies the AP property. 

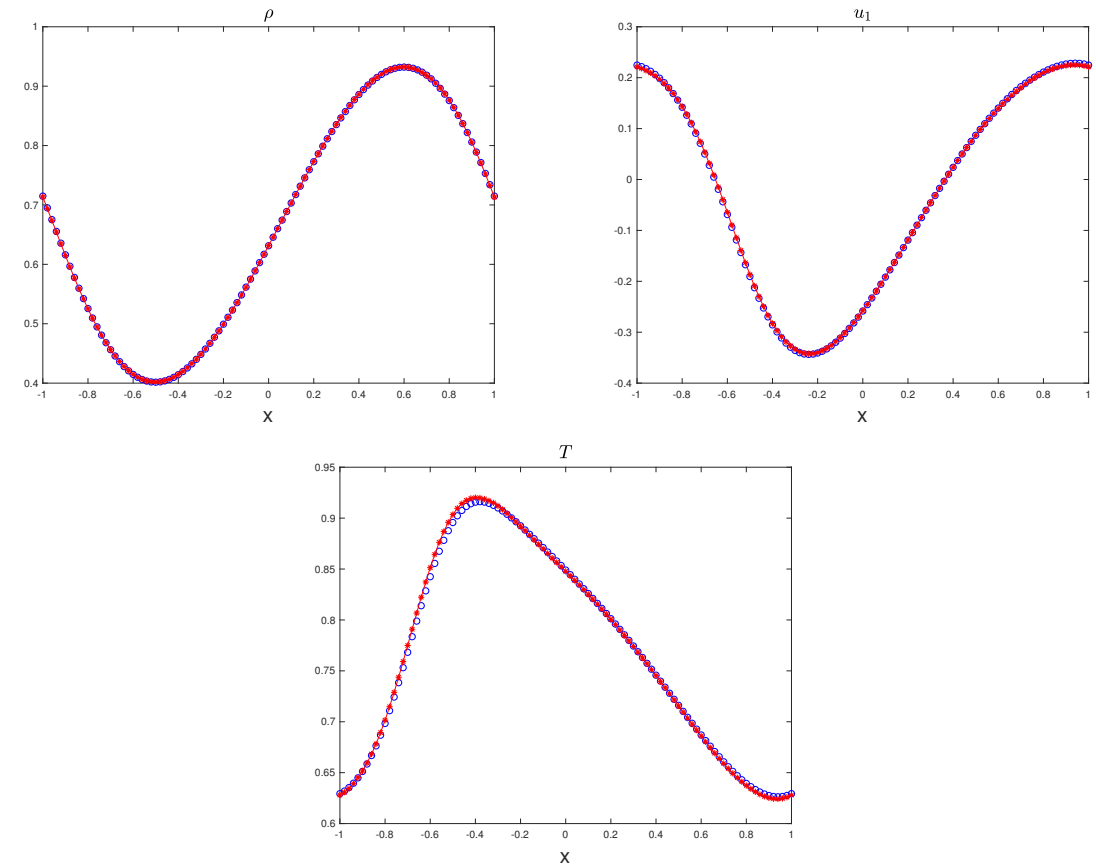

Figure 7: Test II (a). Numerical solutions by 'DS' (circle) and 'MM' (asteroid). $\varepsilon=1, t=0.25$.
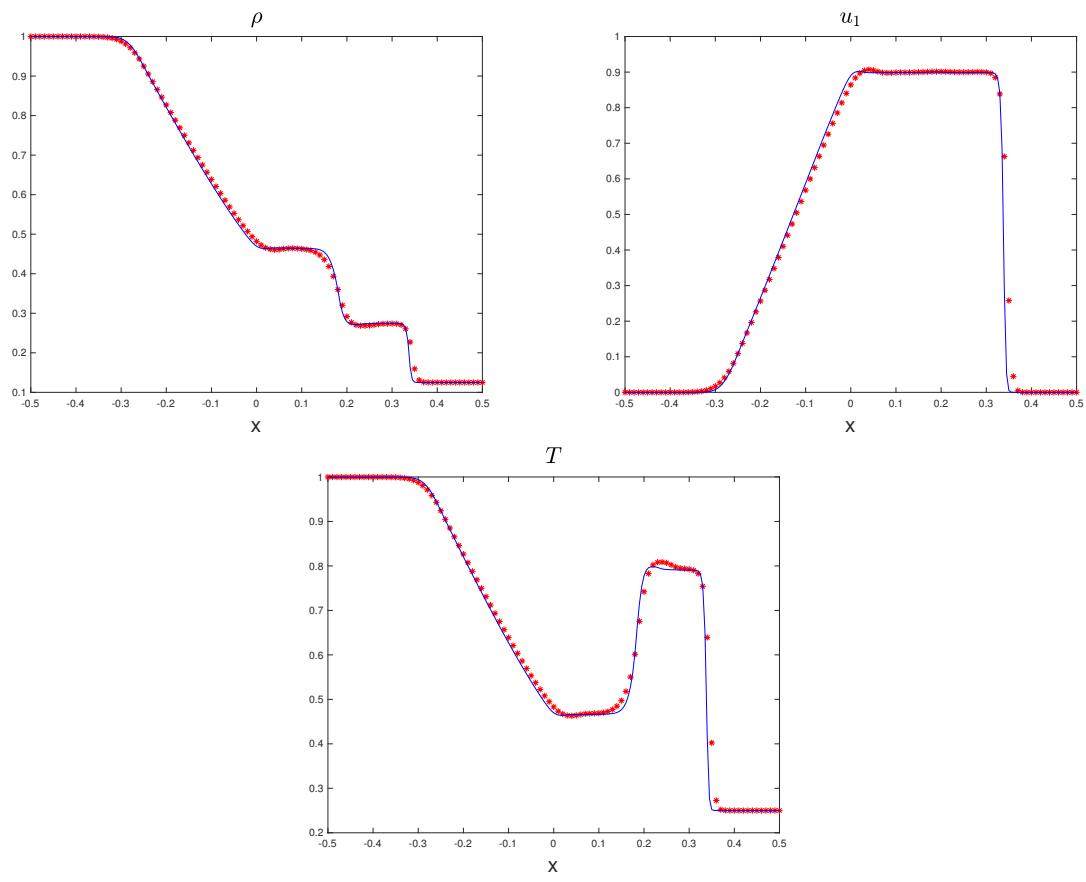

Figure 8: Test II (b). Numerical solutions by 'MM' (asteroid) and reference solutions by using a fine mesh of 'MM' (solid line). $\varepsilon=10^{-3}, t=0.2$. 


\section{A Conservative Scheme for the Vlasov-Ampère- Boltzmann system}

In order to further elaborate the issue of numerical conservative of moments in kinetic solvers, in this section, we develop a conservative scheme for the Vlasov-Ampère-Boltzmann system with or without the collisional term, which is not only of interest for the systems under study, but also gives a general guidance on how to obtain numerically the exact conservation of moments for a general kinetic solver.

\subsection{The collisionless Vlasov-Poisson and Vlasov-Ampère sys- tems}

First, we consider the Vlasov-Poisson (VP) system without collisions between particles,

$$
\left\{\begin{array}{l}
\partial_{t} f+v \cdot \nabla_{x} f-E \cdot \nabla_{v} f=0, \\
\nabla_{x} \cdot E=c(x)-\int_{\mathbb{R}^{d}} f d v .
\end{array}\right.
$$

Here $E$ is the electric field, while $c(x)$ is the background density. The domain is given by $\Omega_{x, v}=\Omega \times \mathbb{R}^{d}$. This system arises in modeling collisionless plasmas 22 . For simplicity, we will always assume periodic boundary condition in $x$ for $f$. Denote the moments as

$$
\rho=\int_{\mathbb{R}^{d}} f d v, \quad \rho u=\int_{\mathbb{R}^{d}} v f d v, \quad E_{\mathrm{Kin}}=\int_{\mathbb{R}^{d}} \frac{1}{2}|v|^{2} f d v .
$$

Moment equations for 7.1 are given by

$$
\left\{\begin{array}{l}
\partial_{t} \rho+\nabla_{x} \cdot(\rho u)=0 \\
\partial_{t}(\rho u)+\nabla_{x} \cdot \int_{\mathbb{R}^{d}} v \otimes v f d v+E \rho=0, \\
\partial_{t} \int_{\mathbb{R}^{d}} \frac{1}{2}|v|^{2} f d v+\nabla_{x} \cdot \int_{\mathbb{R}^{d}} \frac{|v|^{2}}{2} v f d v+E \cdot(\rho u)=0 .
\end{array}\right.
$$

It is easy to check that the system (7.1) conserves the total energy defined

$$
E_{\text {Total }}=\frac{1}{2} \int_{\Omega} \int_{\mathbb{R}^{d}}|v|^{2} f d v d x+\frac{1}{2} \int_{\Omega}|E|^{2} d x
$$

While there have been previous works to develop schemes that conserve this total energy, for example see [3, 4], our strategy is different, and it serves the purpose for a generic strategy to develop energy conserving schemes for collisional system, see the next section. We also refer to [29] for Discontinuous Galerkin solvers for the Boltzmann-Poisson system.

In order to construct a scheme that conserves $E_{\text {Total }}$, we solve the following VlasovAmpè̀re (VA) system by adopting the Ampère's law, instead of solving the Vlasov-Poisson system (7.1,

$$
\begin{aligned}
& \partial_{t} f+v \cdot \nabla_{x} f-E \cdot \nabla_{v} f=0, \\
& \partial_{t} E=\rho u
\end{aligned}
$$

Note that the VA and VP systems are equivalent when the charge solves the continuity equation

$$
\partial_{t} \rho+\nabla_{x} \cdot \rho u=0
$$


Step 1. Update $f^{n+1}$ by solving 7.3 explicitly, that is,

$$
f^{n+1}=f^{n}-\Delta t\left(v \cdot \nabla_{x} f^{n}+E^{n} \cdot \nabla_{v} f^{n}\right) .
$$

Here the transport term $v \cdot \nabla_{x} f$ is approximated by a non-oscillatory high resolution shockcapturing method, and a spectral discretization in the velocity space is used for the term $E \cdot \nabla_{v} f$.

Step 2. Update $E^{n+1}$ by using a forward Euler solver of 7.4,

$$
E^{n+1}=E^{n}+\Delta t(\rho u)^{n}
$$

Step 3. Update the moments at $t^{n+1}$ by solving equations 7.2 and using $f^{n}$.

$$
\left\{\begin{array}{l}
\frac{\rho^{n+1}-\rho^{n}}{\Delta t}+\nabla_{x} \cdot \int_{\mathbb{R}^{d}} v f^{n} d v=0, \\
\frac{(\rho u)^{n+1}-(\rho u)^{n}}{\Delta t}+\nabla_{x} \cdot \int_{\mathbb{R}^{d}} v \otimes v f^{n} d v+E^{n} \cdot \rho^{n}=0, \\
\frac{E_{\mathrm{Kin}}^{n+1}-E_{\mathrm{Kin}}^{n}}{\Delta t}+\nabla_{x} \cdot \int_{\mathbb{R}^{d}} \frac{|v|^{2}}{2} v f^{n} d v+\frac{E^{n}+E^{n+1}}{2} \cdot(\rho u)^{n}=0 .
\end{array}\right.
$$

Theorem 7.1. Let $\left(\rho, u, E_{k i n}, E\right)_{i}$ be the numerical approximation of the corresponding quantities at grid point $x_{i}$. If one discretizes the divergence term in (7.7) by a conservative spatial discretization, then one has the conservations of total mass and energy

$$
\sum_{i=0}^{N_{x}} \rho_{i}^{n+1}=\sum_{i=0}^{N_{x}} \rho_{i}^{n}, \quad \sum_{i=0}^{N_{x}}\left(\left(E_{\text {Kin }}^{n+1}\right)_{i}+\frac{1}{2}\left(E_{i}^{n+1}\right)^{2}\right)=\sum_{i=0}^{N_{x}}\left(\left(E_{\text {Kin }}^{n}\right)_{i}+\frac{1}{2}\left(E_{i}^{n}\right)^{2}\right) .
$$

Proof. Sum over all $i$ for the spatial discretized system of the first equation in (7.7) gives

$$
\Delta x \sum_{i=0}^{N_{x}} \rho_{i}^{n+1}=\Delta x \sum_{i=0}^{N_{x}} \rho_{i}^{n}
$$

Also the third equation of (7.7), after using (7.6), gives

$$
\Delta x \sum_{i=0}^{N_{x}} \frac{\left(E_{\mathrm{Kin}}\right)_{i}^{n+1}-\left(E_{\mathrm{Kin}}\right)_{i}^{n}}{\Delta t}+\frac{1}{2 \Delta t} \sum_{i=0}^{N_{x}} \sum_{i=0}^{N_{x}}\left(E_{i}^{n+1}+E_{i}^{n}\right)(\rho u)_{i}^{n}=0 .
$$

Using (7.6, , one obtains 7.8.

Since the goal of this section is to preserve the total energy in time, we will only conduct numerical examples to check the conservation property, and not consider other discretization issues for the system.

\section{Test III}

Let the initial data be

$$
f(t=0, x, v)=(1+\cos (2 x)) \frac{e^{-|v|^{2} / 2}}{\sqrt{2 \pi}} .
$$

Periodic boundary condition in space is assumed for $f, E$ and $\phi$. The initial condition of the electric field $E$ can be obtained from the Poisson equation

$$
-\Delta_{x} \phi=c(x)-\int_{\mathbb{R}^{d}} f d v
$$


by using a second-order finite-difference Poisson solver and central difference spacial discretization for $E=-\nabla_{x} \phi$. To make the solution unique, we also set the boundary data for $\phi$,

$$
\phi\left(x_{L}\right)=\phi\left(x_{R}\right)=0
$$

Set $c(x)=1$.

Let $x \in[0, \pi], v \in[-2 \pi, 2 \pi], N_{x}=200, N_{v}=64$ and $\Delta t=\Delta x / 20$ in the following test. In Figure 9, the first figure shows the density $\rho(x)$ at time $t=0.5$, computed from either solving the moment equations ('ME') or from the solution $f$ ('Mf'). In the second figure, the electric field $E(x)$ is compared between using the Poisson equation or the Ampére's Law. In the third figure, we plot mass as a function of time and compare it between using ' $\mathrm{ME}$ ' and 'Mf'. One can see that the two solutions match well in the first three figures. In the fourth figure, the total energy, which is obtained from solving the Vlasov-Poisson ('Mf-Poiss'), Vlasov-Ampére system ('Mf-Amp'), or the moment equations and the Ampére's Law ('MEAmp') respectively. This verifies the proof shown in 7.8 that the numerical total energy is perfectly conserved for 'ME-Amp'. The other two lines of 'Mf-Poiss' and 'Mf-Amp', though non-conserved, has a small numerical error (in the order of numerical consistency error). However, it is remarkable to observe that the numerical total energy has an $O\left(10^{-3}\right)$ error for long time which is exactly the same order of magnitude of the numerical total error in the simulation of the Vlasov-Poisson-Landau system computed by means of operator splitting of a DG scheme for the collisionless Vlasov-Poisson advection coupled to the collisional conservative step in Figure 12 of [34, under the same boundary conditions as assumed here. 

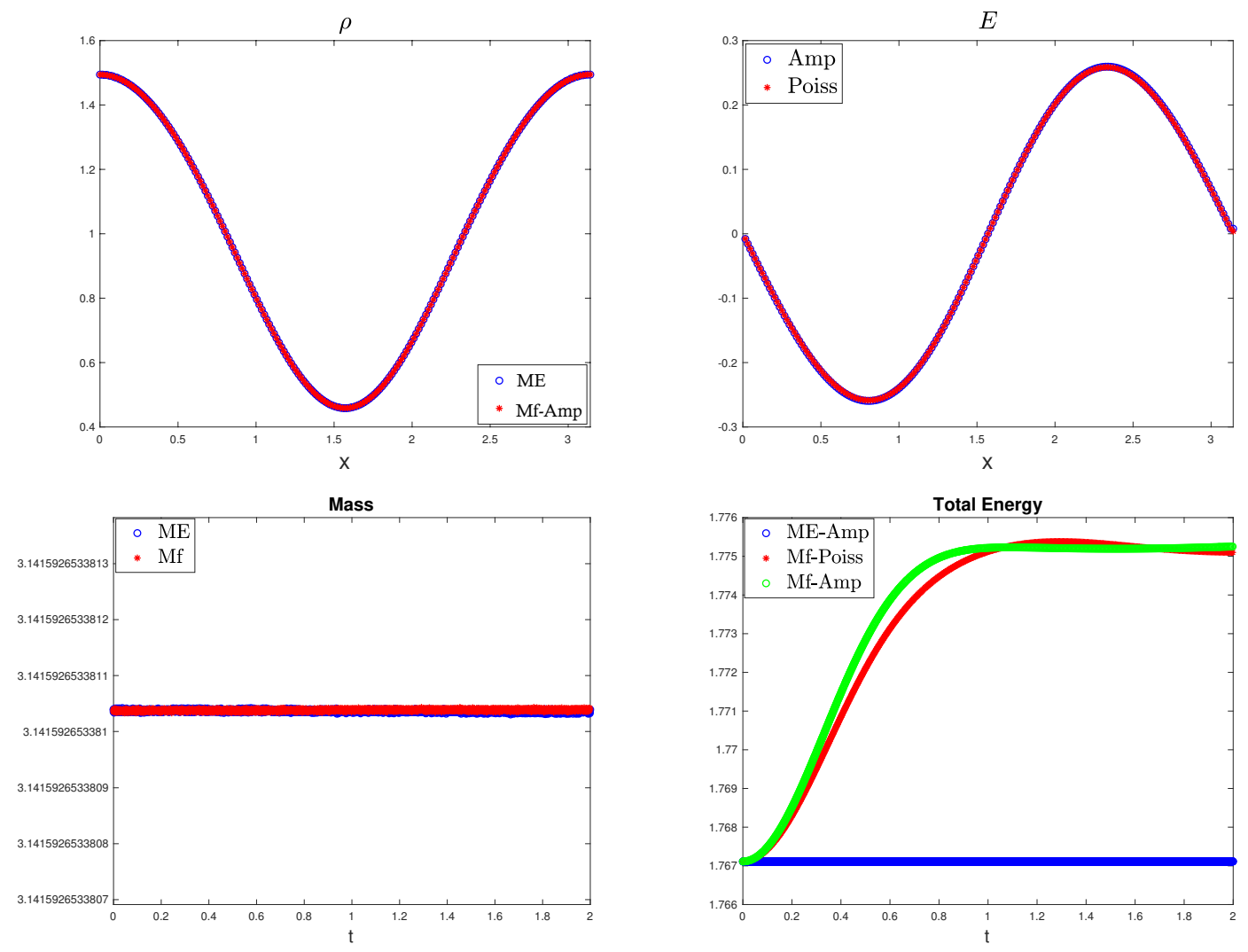

Figure 9: Test III. $\rho, E$ at $t=0.5$ in the first row; mass and total energy with respect to time in the second row.

\subsection{The Vlasov-Ampére-Boltzmann system}

We can easily extend the scheme introduced in section 7.1 to the collisional problems, for example the Vlasov-Ampére-Boltzmann system that will be studied in this section. This system models collisional plasma [17.

Consider the Vlasov-Ampére-Boltzmann system,

$$
\left\{\begin{array}{l}
\partial_{t} f+v \cdot \nabla_{x} f-E \cdot \nabla_{v} f=\frac{1}{\varepsilon} \mathcal{Q}_{\mathrm{B}}(f, f), \\
\partial_{t} E=\rho u .
\end{array}\right.
$$

The time-discretized scheme for the moments equations of 7.11 are the same as the Vlasov-Poisson and is given in 7.2 . With $\rho, \rho u$ and $E_{\text {Kin }}$, one can get the temperature $T$ using the relation $E_{\text {Kin }}=\frac{1}{2} \rho u^{2}+\frac{N_{d}}{2} \rho T$ and thus compute the local equilibrium

$$
M_{\mathrm{eq}}(x, v)=\frac{\rho(x)}{(2 \pi T(x))^{N_{d} / 2}} \exp \left(-\frac{(v-u(x))^{2}}{2 T(x)}\right) .
$$

To overcome the stiffness of the collision operator in the fluid regime, we simply use the Filbet-Jin penalty AP schemes here.

Step 2 and Step 3 given by (7.6) and $(7.7)$ to update $E$ and the moments quantities are exactly the same as the scheme given in section 7.1. With the collision term in 7.11, step 
1 correspondingly becomes

$$
\frac{f^{n+1}-f^{n}}{\Delta t}+v \cdot \nabla_{x} f^{n}-E^{n} \cdot \nabla_{v} f^{n}=\frac{\mathcal{Q}\left(f^{n}\right)-P\left(f^{n}\right)}{\varepsilon}+\frac{P\left(f^{n+1}\right)}{\varepsilon},
$$

which gives

$f^{n+1}=\frac{\varepsilon}{\varepsilon+\beta \Delta t}\left(f^{n}-\Delta t v \cdot \nabla_{x} f^{n}+\Delta t E^{n} \cdot \nabla_{v} f^{n}\right)+\Delta t \frac{\mathcal{Q}\left(f^{n}\right)-P\left(f^{n}\right)}{\varepsilon+\beta \Delta t}+\frac{\beta \Delta t}{\varepsilon+\beta \Delta t} \mathcal{M}^{n+1}$, with $\mathcal{M}^{n+1}$ defined through the moments quantities solved from 7.7.

In the following numerical experiments we use $\Delta x=\pi / 200, \Delta t=\Delta x / 20$.

In Figure 10, we show a similar set of figures as Figure 9 above. The first row shows the numerical solution at output time $t=0.5$, with $\varepsilon=1$. The numerical solutions such as $\rho, E$ match well no matter whether the Ampére's Law or the Poisson equation is used. In this test, moments (mass and total energy) are perfectly conserved if obtained from 'ME' or 'ME-Amp', as shown in the second row of Figure 11 The red line in the third figure indicates that the mass obtained from $f$ is not perfectly conserved but has a spectrally small error. The green ('Mf-Amp') and red ('Mf-Poiss') lines in the fourth figure show that the energy, if obtained from $f$ coupled with the Ampére's Law or the Poisson equation for $E$, is not perfectly conserved but still have a small error.

For the last test, we will only use the exactly conservative scheme and check the penalty method for the Vlasov-Ampére-Boltzmann equation, for the case of small $\varepsilon$. Figure 11 shows in the first row the numerical solution $\rho, E$ at output time $t=0.1$, with $\varepsilon=0.05$. In the second row, we show that mass and total energy are perfectly conserved if using the moments equations given by 7.7 . 

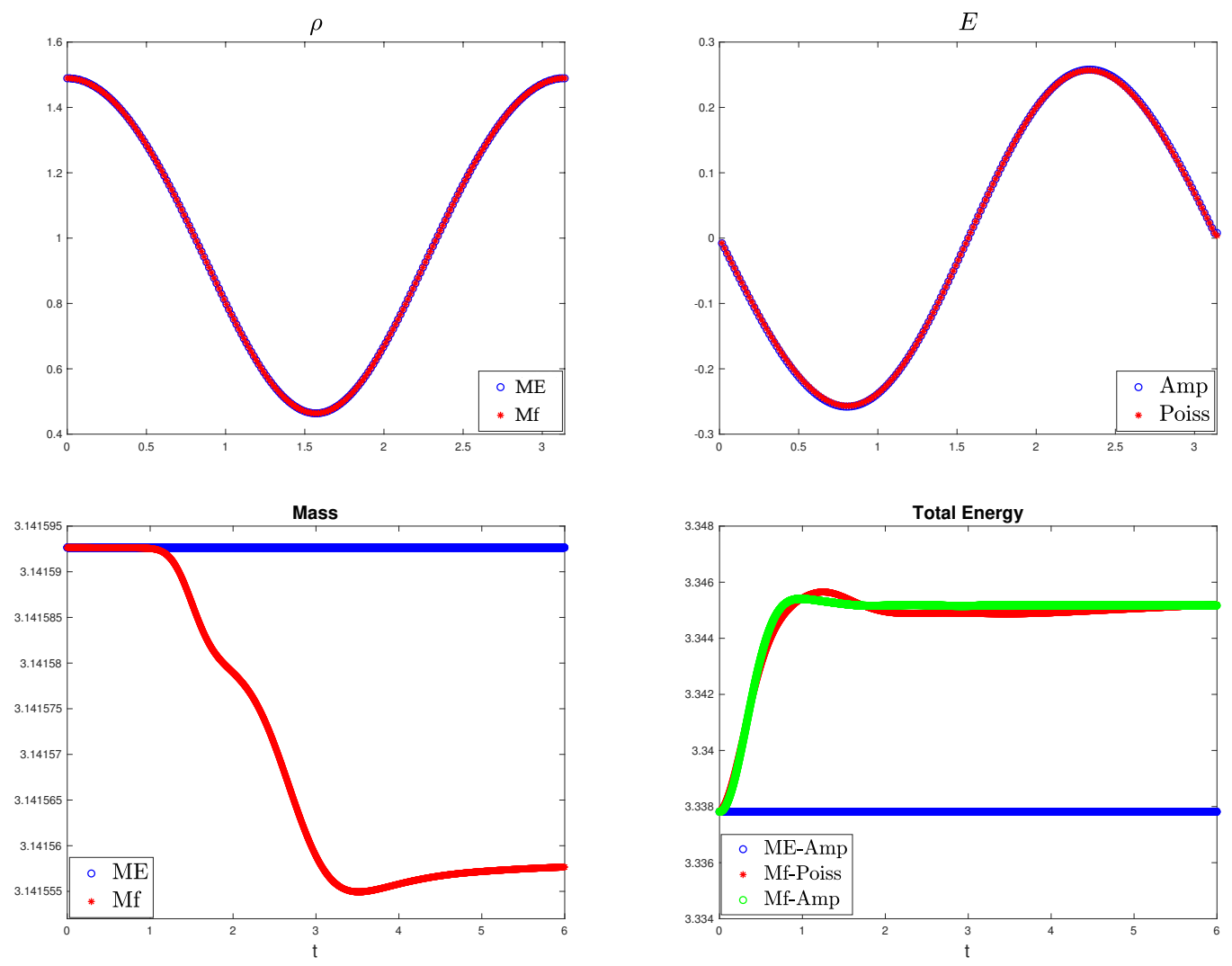

Figure 10: $\varepsilon=1$. Numerical solutions $\rho, E$ at $t=0.5$ in the first row; mass and total energy with respect to time in the second row. 

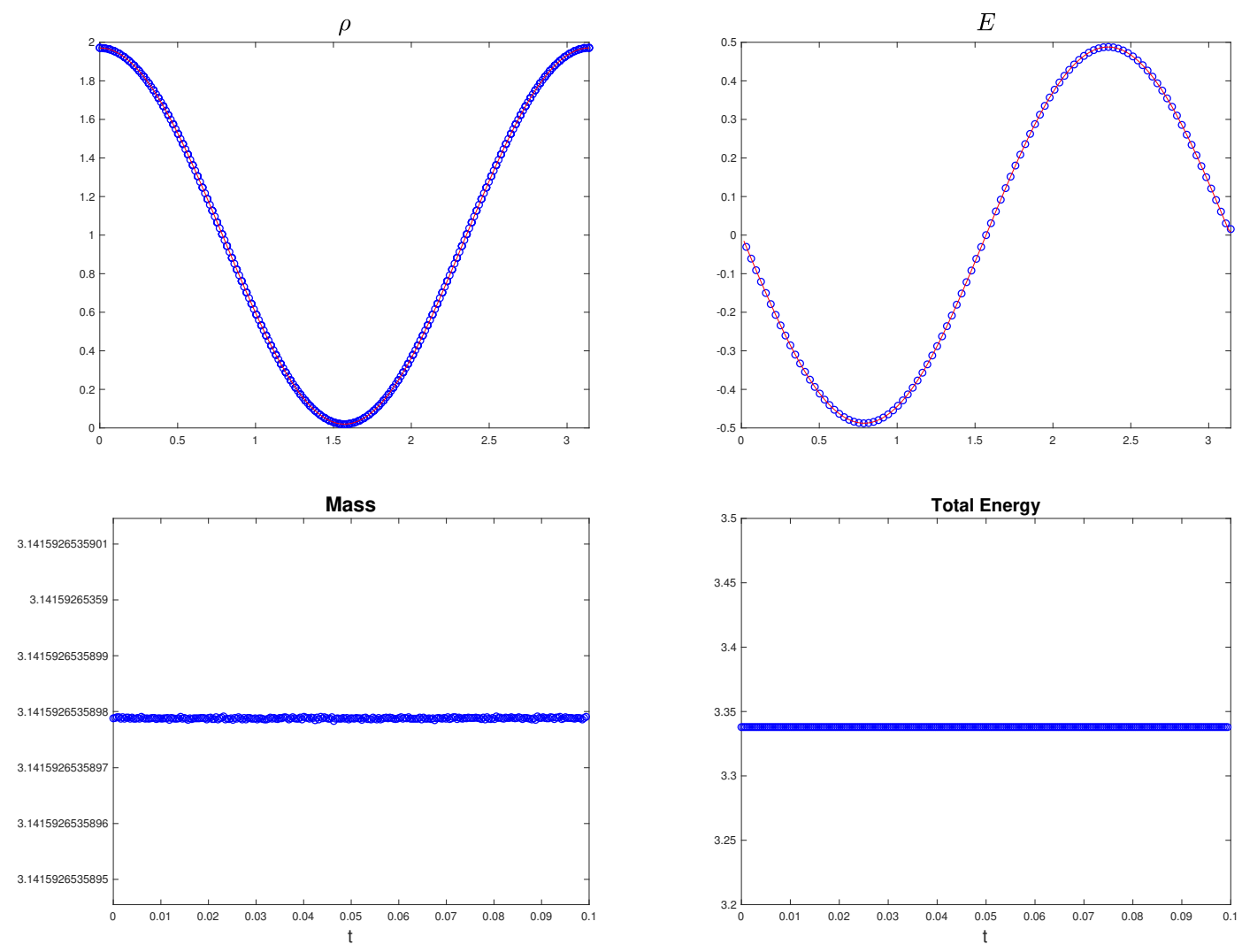

Figure 11: $\varepsilon=0.05$. Numerical solutions $\rho, E$ of the Vlasov-Ampére-Boltzmann equation at $t=0.1$ in the first row (blue circle uses $N_{x}=100, \Delta t=\Delta x / 20$, red line is as reference solution using fine mesh $N_{x}=200, \Delta t=10^{-4}$ ); mass and total energy with respect to time in the second row.

Remark 7.2. The schemes proposed in this section give the desired conservation property thanks to the use of moment equations. Here we obtain the moment system first (so the right hand side vanishes) and then discretize it. If one obtains the moments from the discretized $f$ equation, due to the non-conservation of the approximate collision operator, the discrete moments are not necessarily conserved. This has already been addressed in [15] for a different purpose, but here it serves the purpose as a generic strategy to devise conservative schemes for general collision kinetic system. The only price paid is the extra effort to solve the moment system.

\section{Conclusions and future work}

The micro-macro decomposition based method for multiscale kinetic equations has found many applications as an effective method to derive Asymptotic-Preserving schemes that work efficiently in all regimes, including both the kinetic and fluid regimes. However, so far it has been developed only for the BGK model. In this paper we extend it to general collisional kinetic equations, including the Boltzmann and the Fokker-Planck Landau equations. One 
of the difficulty in this formulation is the numerical stiff linearized collision operator, which needs to be treated implicitly thus becomes numerically difficult. Our main idea is to use a relation between the (numerically stiff) linearized collision operator with the nonlinear quadratic ones, the latter's stiffness can be overcome using the BGK penalization method of Filbet and Jin for the Boltzmann, or the linear Fokker-Planck penalization method of Jin and Yan for the Fokker-Planck Landau equations. Such a scheme allows the computation of multiscale collisional kinetic equations efficiently in all regimes, including the fluid regime in which the fluid dynamic behavior can be correctly computed even without numerically resolving the small Knudsen number. It is implicit but can be implemented explicitly.

This scheme preserves the moments (mass, momentum and energy) exactly due to the use of the macroscopic system which is naturally in a conservative form. We then utilize this conservation property for more general kinetic equations, using the Vlasov-Ampère and Vlasov-Ampère-Boltzmann systems as examples. The main idea is to evolve both the kinetic equation for the probability density distribution and the moment system, the later naturally induces a scheme that conserves exactly the moments numerically if they are physically conserved. This recipe is generic and applies to all kinetic equations.

Numerical examples demonstrate the conservation properties of our schemes, as well as it robustness in the fluid dynamic and mixed regimes. Notice that the numerical total energy exhibited an $O\left(10^{-3}\right)$ error persistent for long time that coincides with the order of magnitude of the numerical total energy error in the implementation of the Vlasov-Poisson-Landau system by operator splitting of a DG scheme for the collisionless Vlasov-Poisson advection coupled to the collisional conservative step in 34. This observation opens an interesting problem of understanding how to diminish this computational error on obtaining the total energy evolution from the kinetic pdf that solves the Vlasov-Poisson with either Boltzmann or Landau collisional forms, by perhaps either imposing a conservation constraint in the kinetic simulation of our proposed scheme or to address operator splitting improvements in the approach used in 34 .

In the numerical simulation, we use a second order space discretization and a first order IMEX temporal discretization. It would be nice to improve the first order time approximation and develop a fully second order scheme, for example, by adopting the method introduced in 32. This will be done in a future work. To extend the micro-macro method for multidimensional problems also remain to be pursued. Here one needs to extend the staggered grid to higher dimension, a task that was investigated for hyperbolic systems of conservative laws [1] but yet to be studied for kinetic equations.

\section{Acknowledgement}

The authors would like to thank both referees for their helpful comments to improve this paper.

\section{Appendix: Details of Numerical Implementation}

Details of solving 4.2 are shown below. In the case of $x \in \mathbb{R}, v \in \mathbb{R}^{2}(d=2)$,

$$
u_{1}=\frac{1}{\rho} \int_{\mathbb{R}^{d}} f v_{1} d v, \quad u_{2}=\frac{1}{\rho} \int_{\mathbb{R}^{d}} f v_{2} d v .
$$


We then have

$$
\begin{aligned}
& \frac{\partial \rho}{\partial t}+\partial_{x} F_{1}=-\varepsilon \partial_{x}\langle g\rangle, \\
& \frac{\partial}{\partial t}\left(\rho u_{1}\right)+\partial_{x} F_{2}=-\varepsilon \partial_{x}\left\langle v_{1}^{2} g\right\rangle, \\
& \frac{\partial}{\partial t}\left(\rho u_{2}\right)+\partial_{x} F_{3}=-\varepsilon \partial_{x}\left\langle v_{1} v_{2} g\right\rangle, \\
& \frac{\partial E}{\partial t}+\partial_{x} F_{4}=-\varepsilon \partial_{x}\left\langle v_{1} \frac{|v|^{2}}{2} g\right\rangle,
\end{aligned}
$$

where

$$
F_{1}=\left\langle v_{1} M\right\rangle, \quad F_{2}=\left\langle v_{1}^{2} M\right\rangle, \quad F_{3}=\left\langle v_{1} v_{2} M\right\rangle, \quad F_{4}=\left\langle v_{1} \frac{|v|^{2}}{2} M\right\rangle .
$$

with $M$ associated with $\rho, u_{1}, u_{2}, T$ as defined in 2.11.

The kinetic formulation of the flux splitting 5.3 is given by

$$
F_{1}^{ \pm}=\left\langle v_{1}^{ \pm} M\right\rangle, \quad F_{2}^{ \pm}=\left\langle v_{1}^{ \pm} v_{1} M\right\rangle, \quad F_{3}^{ \pm}=\left\langle v_{1}^{ \pm} v_{2} M\right\rangle, \quad F_{4}^{ \pm}=\left\langle v_{1}^{ \pm} \frac{|v|^{2}}{2} M\right\rangle,
$$

with $v_{1}^{ \pm}=\left(v_{1} \pm\left|v_{1}\right|\right) / 2, v_{2}^{ \pm}=\left(v_{2} \pm\left|v_{2}\right|\right) / 2$.

\section{References}

[1] C. Bardos, F. Golse, And D. Levermore, Fluid dynamic limits of kinetic equations. I. Formal derivations, J. Statist. Phys., 63 (1991), pp. 323-344.

[2] M. Bennoune, M. Lemou, And L. Mieussens, Uniformly stable numerical schemes for the Boltzmann equation preserving the compressible Navier-Stokes asymptotics, J. Comput. Phys., 227 (2008), pp. 3781-3803.

[3] Y. Cheng, A. Christlieb, And X. Zhong, Numerical study of the two-species VlasovAmpère system: Energy-conserving schemes and the current-driven ion-acoustic instability, Journal of Computational Physics, 288 (2014).

[4] Y. Cheng, A. J. Christlieb, And X. Zhong, Energy-conserving Discontinuous Galerkin methods for the Vlasov-Ampère system, J. Comput. Phys., 256 (2014), pp. 630655.

[5] G. Dimarco and L. Pareschi, Exponential Runge-Kutta methods for stiff kinetic equations, SIAM Journal on Numerical Analysis, 49 (2011), pp. 2057-2077.

[6] F. Filbet And S. Jin, A class of asymptotic-preserving schemes for kinetic equations and related problems with stiff sources, J. Comput. Phys., 229 (2010), pp. 7625-7648.

[7] I. M. GAmBA AND J. R. HAACK, A conservative spectral method for the Boltzmann equation with anisotropic scattering and the grazing collisions limit, Journal of Computational Physics, 270 (2014), pp. 40-57.

[8] I. M. Gamba, J. R. HaAck, C. D. Hauck, and J. Hu, A fast spectral method for the Boltzmann collision operator with general collision kernels, SIAM Journal on Scientific Computing, 39 (2017), pp. B658-B674. 
[9] I. M. Gamba and S. H. Tharkabhushanam, Spectral-Lagrangian methods for collisional models of non-equilibrium statistical states, Journal of Computational Physics, 228 (2009), pp. 2012-2036.

[10] J. JAnG, F. LI, J.-M. QIU, AND T. XIOnG, Analysis of asymptotic preserving DGIMEX schemes for linear kinetic transport equations in a diffusive scaling, SIAM J. Numer. Anal., 52 (2014), pp. 2048-2072.

[11] G.-S. JiAng AND E. TADMOR, Non-oscillatory central schemes for multidimensional hyperbolic conservation laws, SIAM Journal on Scientific Computing, 19 (1998), pp. 18921917.

[12] S. JIN, Efficient asymptotic-preserving (AP) schemes for some multiscale kinetic equations, SIAM Journal on Scientific Computing, 21 (1999), pp. 441-454.

[13] —- Asymptotic preserving (AP) schemes for multiscale kinetic and hyperbolic equations: a review, Lecture notes for summer school on methods and models of kinetic theory (M\&MKT), Porto Ercole (Grosseto, Italy), (2010), pp. 177-216.

[14] S. Jin AND Q. LI, A BGK-penalization-based asymptotic-preserving scheme for the multispecies Boltzmann equation, Numer. Methods Partial Differential Equations, 29 (2013), pp. 1056-1080.

[15] S. Jin AND B. YAN, A class of asymptotic-preserving schemes for the Fokker-PlanckLandau equation, J. Comput. Phys., 230 (2011), pp. 6420-6437.

[16] A. KlaR AND C. SChmeiser, Numerical passage from radiative heat transfer to nonlinear diffusion models, Mathematical Models and Methods in Applied Sciences, 11 (2001), pp. $749-767$.

[17] N. Krall And A. TRIVElPIECE, Principles of plasma physics. international series in pure and applied physics, 1973.

[18] M. Lemou, Relaxed micro-macro schemes for kinetic equations, C. R. Math. Acad. Sci. Paris, 348 (2010), pp. 455-460.

[19] M. Lemou And L. Mieussens, A new asymptotic preserving scheme based on micromacro formulation for linear kinetic equations in the diffusion limit, SIAM J. Sci. Comput., 31 (2008), pp. 334-368.

[20] R. J. LeVEque, Numerical methods for conservation laws, Lectures in Mathematics ETH Zürich, Birkhäuser Verlag, Basel, second ed., 1992.

[21] Q. Li AND L. PARESChi, Exponential Runge-Kutta for the inhomogeneous Boltzmann equations with high order of accuracy, Journal of Computational Physics, 259 (2014), pp. $402-420$.

[22] R. Liboff, Kinetic Theory: Classical, Quantum and Relativistic Descriptions.

[23] C. Liu, K. Xu, Q. Sun, AND Q. CAI, A unified gas-kinetic scheme for continuum and rarefied flows IV: Full Boltzmann and model equations, Journal of Computational Physics, 314 (2016), pp. 305-340. 
[24] J.-G. Liu And L. Mieussens, Analysis of an asymptotic preserving scheme for linear kinetic equations in the diffusion limit, SIAM Journal on Numerical Analysis, 48 (2010), pp. 1474-1491.

[25] T.-P. LiU, T. YAng, S.-H. YU, AND H.-J. ZhaO, Nonlinear stability of rarefaction waves for the Boltzmann equation, Archive for rational mechanics and analysis, 181 (2006), p. 333 .

[26] T.-P. LiU AND S.-H. YU, Boltzmann equation: micro-macro decompositions and positivity of shock profiles, Communications in mathematical physics, 246 (2004), pp. 133-179.

[27] L. Mieussens, Discrete-velocity models and numerical schemes for the Boltzmann-BGK equation in plane and axisymmetric geometries, Journal of Computational Physics, 162 (2000), pp. 429-466.

[28] - On the asymptotic preserving property of the unified gas kinetic scheme for the diffusion limit of linear kinetic models, J. Comput. Phys., 253 (2013), pp. 138-156.

[29] J. Morales Escalante, I. Gamba, A. Majorana, Y. Cheng, C.-W. Shu, And J. Chelikowsky, Discontinuous Galerkin deterministic solvers for a BoltzmannPoisson model of hot electron transport using an averaged empirical pseudopotential band, Comput. Methods Appl. Mech. Engrg, 321 (2017), pp. 209-234.

[30] C. Mouhot And L. PAReschi, Fast algorithms for computing the Boltzmann collision operator, Mathematics of computation, 75 (2006), pp. 1833-1852.

[31] L. PAReschi AND G. Russo, Numerical solution of the Boltzmann equation I: Spectrally accurate approximation of the collision operator, SIAM journal on numerical analysis, 37 (2000), pp. 1217-1245.

[32] - Implicit-Explicit Runge-Kutta schemes and applications to hyperbolic systems with relaxation, J. Sci. Comput., 25 (2005), pp. 129-155.

[33] K. Xu And J.-C. HuAng, A unified gas-kinetic scheme for continuum and rarefied flows, Journal of Computational Physics, 229 (2010), pp. 7747-7764.

[34] C. Zhang And I. M. Gamba, A conservative scheme for Vlasov Poisson Landau modeling collisional plasmas, Journal of Computational Physics, 340 (2017), pp. 470-497. 